\title{
Improving Nuclear Safety of Fast Reactors by Slowing Down Fission Chain Reaction
}

\author{
G. G. Kulikov, A. N. Shmelev, and V. A. Apse \\ National Research Nuclear University MEPhI (Moscow Engineering Physics Institute), Kashirskoe shosse 31, Moscow 115409, Russia \\ Correspondence should be addressed to G. G. Kulikov; ggkulikov@mephi.ru
}

Received 23 May 2014; Revised 19 September 2014; Accepted 20 September 2014; Published 16 October 2014

Academic Editor: Massimo Zucchetti

Copyright (C) 2014 G. G. Kulikov et al. This is an open access article distributed under the Creative Commons Attribution License, which permits unrestricted use, distribution, and reproduction in any medium, provided the original work is properly cited.

Light materials with small atomic mass (light or heavy water, graphite, and so on) are usually used as a neutron reflector and moderator. The present paper proposes using a new, heavy element as neutron moderator and reflector, namely, "radiogenic lead" with dominant content of isotope ${ }^{208} \mathrm{~Pb}$. Radiogenic lead is a stable natural lead. This isotope is characterized by extremely low micro cross-section of radiative neutron capture $(\sim 0.23 \mathrm{mb})$ for thermal neutrons, which is smaller than graphite and deuterium cross-sections. The reflector-converter for a fast reactor core is the structure capable of transforming some part of prompt neutrons leaked from the core into the reflected neutrons with properties similar to those of delayed neutrons, that is, sufficiently large contribution to reactivity at the level of effective fraction of delayed neutrons and relatively long lifetime, comparable with lifetimes of radionuclides-emitters of delayed neutrons. It is evaluated that the use of radiogenic lead makes it possible to slow down the chain fission reaction on prompt neutrons in the fast reactor. This can improve the fast reactor safety and reduce some requirements to the technologies used to fabricate fuel for the fast reactor.

\section{Introduction}

Importance of such physical characteristic as prompt neutron lifetime for nuclear reactor safety is well known for a long time and was reflected in numerous publications, for example, in one of such fundamental works as [1]. The longer prompt neutron lifetime could produce the most favorable effects on nuclear reactor safety under conditions of the reactivity-induced accidents.

The positive role to be played by the radiogenic lead, that is, lead with a dominant content of isotope ${ }^{208} \mathrm{~Pb}$, as a coolant for fast reactor safety was first noted in works $[2,3]$, where a possibility for significant improvement of the coolant temperature reactivity coefficient was shown. Later, some possibilities for improving other neutron-physical and thermal-hydraulic parameters of power fast reactors were considered at usage of the radiogenic lead as a coolant [4]. This direction of researches has been developed in work [5], where, in addition to the aforementioned possibilities, the prospects of the radiogenic lead applications for developing high-flux accelerator-driven systems capable to transmute radioactive wastes and for upgrading proliferation resistance of advanced $\mathrm{Pu}$-based fuel compositions were investigated too.

Extension of prompt neutron lifetime in fast reactors with the radiogenic lead as a neutron reflector was first proposed in works $[6,7]$. This paper presents the results of further studies carried out in this direction.

\section{Prompt Neutron Lifetime of Different Reactors}

The longer prompt neutron lifetimes can substantially improve kinetic response of the fast reactor to a jump-like insertion of relatively large $(\sim 1 \$$ or even more) positive reactivity. For simplicity, let effective fraction $\beta$ of delayed neutrons be the same for all the fast reactor models under consideration here, $\beta\left({ }^{235} \mathrm{U}\right)=0.0065$.

The curves presented in Figure 1 demonstrate dependencies of the reactivity jump required to provide the 
power excursion with asymptotic time period $T$ (without any feedback effects) in the fourth-generation lead-cooled, fast BREST-type [8] reactor (neutron reflector $-50 \mathrm{~cm}$ thick natural lead), in thermal VVER-type and CANDU-type reactors as well as in two hypothetical reactors whose prompt neutron lifetimes are equal to $0.01 \mathrm{~s}$ and $0.1 \mathrm{~s}$, respectively.

These curves demonstrate that the shorter lifetime of prompt neutrons results in the faster power excursion at the same reactivity jump. It seems helpful to reformulate the statement as follows. If prompt neutron lifetime became longer, then the power excursion with a certain asymptotic time period $T$ would require the larger reactivity jump. Otherwise, the power excursion will be slower. Consequently, the reactor safety can be enhanced by making lifetime of prompt neutrons longer.

For example, based on one-point model of neutron kinetics, it may be concluded that application of thick ${ }^{208} \mathrm{~Pb}$ reflector in the fast reactor BREST allowed us to reach prompt neutron lifetime of $\sim 1$ ms. If 1 -\$ reactivity jump (!) occurs in the fast reactor, then its power increases with the excursion period nearly $1 \mathrm{~s}$ instead of $14 \mathrm{~ms}$ in the fast reactor reflected by natural lead. If prompt neutron lifetime in the fast reactor BREST reflected by thick ${ }^{208} \mathrm{~Pb}$ layer is prolonged up to $10 \mathrm{~ms}$, then the power excursion period will be longer than $1 \mathrm{~s}$ even at the reactivity jumps up to $2 \$$. So long the power excursion periods give sufficient time for coolant to remove the heat from fuel rods. This means that feedbacks on coolant temperature and coolant density could be apparently actuated.

\section{One-Point Model}

The dependencies shown in Figure 1 were calculated with the application of one-point kinetic model with six groups of delayed neutrons. No feedback effects were taken into consideration. The one-point model is based on the following set of equations:

$$
\begin{gathered}
\frac{d n(t)}{d t}=\frac{\rho-\beta}{\bar{\Lambda}_{\mathrm{prt}}} \cdot n(t)+\sum_{i=1}^{6} \lambda_{i} \cdot C_{i}(t), \\
\frac{d C_{i}(t)}{d t}=\frac{\beta_{i}}{\bar{\Lambda}_{\mathrm{prt}}} \cdot n(t)-\lambda_{i} \cdot C_{i}(t) ; \quad(i=1,2, \ldots, 6),
\end{gathered}
$$

where $n$ is neutron population in the reactor core; $C_{i}$ is quantity of nuclei-emitters for $i$ th group of delayed neutrons in the reactor core; $\rho=\left(k_{\mathrm{ef}}-1\right) / k_{\mathrm{ef}}$, reactivity, where $k_{\mathrm{ef}}$ is the effective neutron multiplication factor in the reactor including neutron reflector; $\beta$ is effective fraction of delayed neutrons; $\beta_{i}$ is effective fraction of delayed neutrons in $i$ th group; $\bar{\Lambda}_{\text {prt }}$ mean prompt neutron lifetime (according to the terminology applied by Bell and Glasstone [1]) in the reactor including neutron reflector; $\lambda_{i}$ is decay constant of nucleiemitters in $i$ th group of delayed neutrons. Prompt neutron lifetime $\bar{\Lambda}_{\text {prt }}$ and generation time $\tau$ are linked by the relationship: $\tau=\bar{\Lambda}_{\text {prt }} / k_{\text {ef }}$. Since only critical andnearly critical

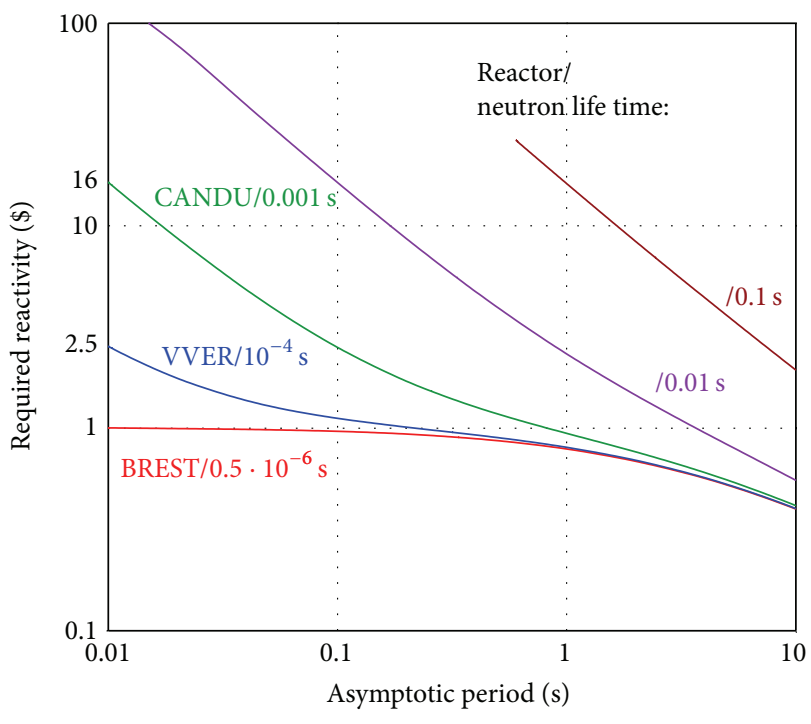

FIGURE 1: Dependencies of the reactivity jump required to provide the power excursion with asymptotic time period $T$ (one-point model, no feedback effects).

(within some dollars from criticality) states are analyzed here, lifetime and generation time differ insignificantly. These terms are regarded below as being equivalent.

Within the frames of one-point kinetic model (1), the inverse-hour equation that links the asymptotic period of the power excursion $T$ with the inserted positive reactivity $\rho$ may be written in the following form:

$$
\rho=\frac{\bar{\Lambda}_{\mathrm{prt}}}{T}+\sum_{i=1}^{6} \frac{\beta_{i}}{1+\lambda_{i} \cdot T} .
$$

\section{Two-Point Model}

As has been noted in [9], one-point model is not completely applicable for analysis of neutron kinetics in the reactor cores surrounded by neutron reflector. The associated problems were analyzed in [9-15] with application of multipoint models or with introduction of some additional (fictive) groups of delayed neutrons. The latter case takes into account the following new parameters: time of neutron staying in the reactor core before leakage, time for neutron transport from the reactor core to neutron reflector, time of neutron staying in the reflector, time for neutron transport from the reflector into the reactor core, and time from neutron arrival into the reactor core to initiation of fission reaction by this neutron. In the case under consideration here, neutron reflector is placed in the immediate vicinity to the reactor core, and the reflector is thick from physical point of view. This means that time of neutron staying in the reflector plays a dominant role in competition with all other times. If one additional group of delayed neutrons, namely, slow neutrons coming back from the reflector into the reactor core, is introduced to study 
the reflector-induced effects on neutron kinetics; then, the following set of equations may be written:

$$
\begin{gathered}
\frac{d n(t)}{d t}=\frac{\rho-\beta-\rho_{R}}{\bar{\Lambda}_{C}} \cdot n(t)+\sum_{i=1}^{6} \lambda_{i} \cdot C_{i}(t)+\lambda_{R} \cdot C_{R}(t), \\
\frac{d C_{i}(t)}{d t}=\frac{\beta_{i}}{\bar{\Lambda}_{C}} \cdot n(t)-\lambda_{i} \cdot C_{i}(t) ; \quad(i=1,2, \ldots, 6), \\
\frac{d C_{R}(t)}{d t}=\frac{\rho_{R}}{\bar{\Lambda}_{C}} \cdot n(t)-\lambda_{R} \cdot C_{R}(t) ; \quad \lambda_{R}=\frac{1}{\bar{\Lambda}_{R}},
\end{gathered}
$$

where, in addition to the aforementioned designations, $C_{R}$ is quantity of fictive nuclei-emitters for additional group of delayed neutrons, that is, for neutrons coming back from the reflector into the reactor core, where they can initiate fission reactions (for brevity, $C_{R}$ may be called a quantity of fictive nuclei-emitters in the reflector); $\rho_{R}=\left(k_{\mathrm{ef}}-k_{\mathrm{ef}}^{\mathrm{C}}\right) / k_{\mathrm{ef}}$ is reactivity gain caused by the reflector, that is, increase of reactivity caused by neutrons coming back from the reflector into the reactor core and subsequent initiation of additional fission reactions (for brevity, $\rho_{R}$ may be called as a reactivity gain caused by the reflector); $k_{\text {ef }}$ is effective neutron multiplication factor in the reactor, including neutron reflector (like in one-point model); $k_{\mathrm{ef}}^{C}$ is effective neutron multiplication factor in the reactor without neutron reflector; $\bar{\Lambda}_{C}$ is prompt neutron lifetime in the reactor without neutron reflector; $\bar{\Lambda}_{R}$ is prompt neutron lifetime caused by the reflector-induced effects; that is, $\bar{\Lambda}_{R}$ is a sum of neutron lifetime in the reactor core, time of neutron staying in the reflector and neutron lifetime in the reactor core after coming back from the reflector (for brevity, $\bar{\Lambda}_{R}$ may be called as a prompt neutron lifetime in the reflector).

According to the set of equations (3), additional group of delayed neutrons, which simulates neutron diffusion in the reflector, can be characterized by its effective fraction, that is, by contribution of the reflector into reactivity and by its decay constant, that is, inverse value of prompt neutron lifetime in the reflector. The inverse-hour equation that links the asymptotic period $T$ with the inserted positive reactivity $\rho$ can be written in the following form for the reactor core surrounded by neutron reflector:

$$
\rho=\frac{\bar{\Lambda}_{C}}{T}+\frac{\rho_{R}}{1+T / \bar{\Lambda}_{R}}+\sum_{i=1}^{6} \frac{\beta_{i}}{1+\lambda_{i} \cdot T} .
$$

The equation differs from similar inverse-hour equation (2) in one-point model (1) by the second summand that describes the role of neutron reflector. Besides, the first summand of the inverse-hour in one-point model contains mean prompt neutron lifetime in the reactor as a whole, including neutron reflector, while the first summand of the inverse-hour equation in the model with additional group of delayed neutrons (two-point model) contains prompt neutron lifetime $\bar{\Lambda}_{C}$ in the reactor core without neutron reflector, and the second summand contains prompt neutron lifetime in the reflector $\bar{\Lambda}_{R}$. Prompt neutron lifetime in the reflector $\bar{\Lambda}_{R}$ can be calculated from the following balance relationship (see derivation of it in Appendix C):

$$
\bar{\Lambda}_{\mathrm{prt}}=\left(1-\rho_{R}\right) \cdot \bar{\Lambda}_{\mathrm{C}}+\rho_{R} \cdot \bar{\Lambda}_{R}
$$

where $\bar{\Lambda}_{\text {prt }}$ (like in one-point model) is a mean prompt neutron lifetime in the reactor with neutron reflector.

The balance relationship defines prompt neutron lifetime in a system as a sum of prompt neutron lifetimes in all system components (in the reactor core plus in the reflector, for instance) with the weighing coefficients that characterize contributions of these components to total criticality.

It may be concluded from (4) that $\rho_{R}$ plays here a role of additional fraction of delayed neutrons that is characterized by $\bar{\Lambda}_{R}$, prompt neutron lifetime in the reflector. This means, in its turn, that application of such a thick neutron reflector gives a new quality to the reactor-the larger fraction of delayed neutrons and, as a consequence, slowing down of chain fission reaction.

One else important circumstance consists in the following fact. The larger fraction of delayed neutrons depends mainly on neutron leakage from the reactor core and, thus, may be chosen as a developer wills, while fraction of nucleiemitters of delayed neutrons may be chosen only within very stringent constraints. Evidently, generation rate of these "delayed" neutrons substantially depends on leakage rate of fast and resonance neutrons from the reactor core. That is why application of thick neutron reflector is a reasonable option not only for fast reactors but also for the reactors with resonance and even thermal spectra, with small sizes of the reactor core, that is, for the reactors with significant leakage of fast and resonance neutrons from the reactor core.

The inverse-hour equation (4) for the reactor core surrounded by neutron reflector with additional group of delayed neutrons coincides with the inverse-hour equation in two-point model [9]. For brevity, hereafter, the term " $n$ point" will be used in designation of kinetic models, changing only the number of "points."

\section{Multipoint Model}

This model of neutron kinetics can be used by considering a neutron reflector as a whole (as one zone in two-point model) or by considering a neutron reflector as a set of annular (nonintersecting) layers (multipoint model depending on the number of these layers):

$$
\begin{aligned}
\frac{d n(t)}{d t}= & \frac{\rho-\beta-\sum_{j=1}^{J} \rho_{R}^{j}}{\bar{\Lambda}_{C}} \cdot n(t)+\sum_{i=1}^{6} \lambda_{i} \cdot C_{i}(t) \\
& +\sum_{j=1}^{J} \lambda_{R}^{j} \cdot C_{R}^{j}(t),
\end{aligned}
$$




$$
\begin{gathered}
\frac{d C_{i}(t)}{d t}=\frac{\beta_{i}}{\bar{\Lambda}_{C}} \cdot n(t)-\lambda_{i} \cdot C_{i}(t) ; \\
(i=1,2, \ldots, 6 ; j=1,2, \ldots, J) ; \\
\frac{d C_{R}^{j}(t)}{d t}=\frac{\rho_{R}^{j}}{\bar{\Lambda}_{C}} \cdot n(t)-\lambda_{R}^{j} \cdot C_{R}^{j}(t) ; \quad \lambda_{R}^{j}=\frac{1}{\Lambda_{R}^{j}} .
\end{gathered}
$$

Within the frames of multipoint model the inverse-hour equation (4) and the balance relationship (5) for determination of prompt neutron lifetime in a neutron reflector can be rewritten as follows:

$$
\begin{gathered}
\rho=\frac{\bar{\Lambda}_{C}}{T}+\sum_{j=1}^{J} \frac{\rho_{R}^{j}}{1+T / \Lambda_{R}^{j}}+\sum_{i=1}^{6} \frac{\beta_{i}}{1+\lambda_{i} \cdot T}, \\
\Lambda_{\mathrm{prt}}^{j+1}=\left(1-\rho_{R}^{j+1}\right) \cdot \Lambda_{\mathrm{prt}}^{j}+\rho_{R}^{j+1} \cdot \Lambda_{R}^{j+1},
\end{gathered}
$$

where $J$ is the number of annular layers in neutron reflector; $\rho_{R}^{j}$ is contribution of $j$ th layer to total reactivity gain; $\Lambda_{R}^{j}$ is prompt neutron lifetime in $j$ th layer, that is, sum of prompt neutrons lifetime in the reactor core before they go to the reflector, time for neutron transport from the reactor core to $j$ th layer of the reflector, time of neutron staying in $j$ th layer, time for neutron transport from $j$ th layer of the reflector to the reactor core, and lifetime of neutrons after they came back from the reflector; $C_{R}^{j}$ is quantity of nuclei-emitters for additional group of delayed neutrons in $j$ th layer of the reflector; $\Lambda_{\text {prt }}^{j}$ is prompt neutron lifetime in the reactor with neutron reflector consisting of the first $j$ layers, adjacent to the reactor core.

The further results were obtained for six-layer reflector (thickness of the first layer is $50 \mathrm{~cm}$, thicknesses of the next layers are equal to $1 \mathrm{~m}$ each). Kinetics parameters such as $\bar{\Lambda}_{C}$ and spatial dependences of $\bar{\Lambda}_{R}$ and $\rho_{R}$ were evaluated on the base of numerical analysis using diffusion neutron transport model with an evaluated nuclear data library (RUSFOND2010) in frames of one-dimension spherical geometry. Contributions of the reflector into the reactivity gain and prompt neutron lifetime are presented in Table 1 for two-point and multipoint models of neutron kinetics in the fast BREST-type reactor with the reactor core surrounded by neutron reflector of different thickness (from $1 \mathrm{~m}$ to $6 \mathrm{~m}$ ). Multipoint model for the case of $1 \mathrm{~m}$-thick reflector is, in essence, two-point model: the first point for the reactor core with $0.5 \mathrm{~m}$-thick reflector, the second point for annular layer (from $0.5 \mathrm{~m}$ to $1 \mathrm{~m})$ in the reflector. If thickness of the reflector increases up to $2 \mathrm{~m}$, then the third point arises for annular layer from $1 \mathrm{~m}$ to $2 \mathrm{~m}$. Three-point model is used in this case. Correspondingly, neutron kinetics in $6 \mathrm{~m}$ thick reflector is defined by sevenpoint model.

It can be seen that, in the case of $6 \mathrm{~m}$ reflector (sevenpoint model), prompt neutron lifetime in the last $1 \mathrm{~m}$ thick reflector layer is considerably longer (about one order of magnitude) than that for two-point model (one point for the reflector as a whole). This means that more correct mathematical models must be used to provide proper accounting for neutron transport effects in the fast reactors surrounded by physically thick and weakly absorbing neutron reflectors.

\section{Spherically Symmetrical Continuous Model of Neutron Kinetics in Reactor Surrounded by Physically Thick Neutron Reflector}

In order to simplify explanation of continuous neutron kinetics model, it seems reasonable to consider a spherically symmetrical reactor with a physically thick neutron reflector. The reactor core is described by one point in the continuous model. When the number of the reflector layers becomes infinite, multipoint model naturally converts into the continuous model where summing operation should be replaced by integration:

$$
\begin{aligned}
& \frac{d n(t)}{d t}=\frac{\rho-\beta-\rho_{R}\left(r_{\text {out }}\right)}{\bar{\Lambda}_{C}} \cdot n(t) \\
& \quad+\sum_{i=1}^{6} \lambda_{i} \cdot C_{i}(t)+\int_{r_{\text {in }}}^{r_{\text {out }}} \lambda_{R}\left(r^{\prime}\right) \cdot C_{R}\left(r^{\prime}, t\right) \cdot d r^{\prime}, \\
& \frac{d C_{i}(t)}{d t}=\frac{\beta_{i}}{\bar{\Lambda}_{C}} \cdot n(t)-\lambda_{i} \cdot C_{i}(t) ; \quad(i=1,2, \ldots, 6), \\
& \frac{d C_{R}(r, t)}{d t}=\frac{d \rho_{R}(r) / d r}{\bar{\Lambda}_{C}} \cdot n(t)-\lambda_{R}(r) \cdot C_{R}(r, t) ; \\
& \lambda_{R}(r)=\frac{1}{\Lambda_{R}(r)},
\end{aligned}
$$

where $r_{\text {in }}$ and $r_{\text {out }}$ are inner and outer radii of the reflector; $\rho_{R}(r)$ is reactivity gain caused by the reflector with outer radius $r\left(r_{\text {in }} \leq r \leq r_{\text {out }}\right) ; d \rho_{R}(r) / d r$ is differential reactivity gain, that is, accretion of the reactivity gain caused by adding spherical reflector layer of unitary thickness to the reflector with outer radius $r ; C_{R}(r, t)$ is quantity of fictive nuclei-emitters for additional group of delayed neutrons in spherical reflector layer of unitary thickness in spatial point with radius-vector $r ; \Lambda_{R}(r)$ is prompt neutron lifetime in spherical reflector layer of unitary thickness in spatial point with radius-vector $r$.

The inverse-hour equation and relationship for determination of prompt neutron lifetime for continuous model of the reflector in spherically symmetrical geometry can be rewritten in the following new forms:

$$
\begin{gathered}
\rho=\frac{\bar{\Lambda}_{C}}{T}+\int_{r_{\text {in }}}^{r_{\text {out }}} \frac{d \rho_{R}\left(r^{\prime}\right) / d r^{\prime}}{1+T / \Lambda_{R}\left(r^{\prime}\right)} \cdot d r^{\prime}+\sum_{i=1}^{6} \frac{\beta_{i}}{1+\lambda_{i} \cdot T} \\
\Lambda_{R}(r)=\Lambda_{\mathrm{prt}}(r)+\frac{d \Lambda_{\mathrm{prt}}(r) / d r}{d \rho_{R}(r) / d r}
\end{gathered}
$$

Continuous model of neutron kinetics may be regarded as a model describing continuous variation of reactivity gain caused by continuous variation of prompt neutron lifetime in the neutron reflector depending on spatial coordinate within the reflector. Solution of the inverse-hour equation (9) is not 
TABLE 1: Dependence of reactivity gain and prompt neutron lifetime on thickness of ${ }^{208} \mathrm{~Pb}$ reflector in the fast BREST-type reactor for various models of neutron kinetics.

\begin{tabular}{|c|c|c|c|c|c|c|}
\hline \multicolumn{7}{|c|}{ Two-point model } \\
\hline Thickness of ${ }^{208} \mathrm{~Pb}$ reflector, $\mathrm{m}$ & 1 & 2 & 3 & 4 & 5 & 6 \\
\hline Reactivity gain caused by the reflector, $\$$ & 3.01 & 5.30 & 6.51 & 7.21 & 7.58 & 7.78 \\
\hline Prompt neutron lifetime in the reflector, s & $2.90 \cdot 10^{-5}$ & $1.04 \cdot 10^{-3}$ & $5.71 \cdot 10^{-3}$ & $1.21 \cdot 10^{-2}$ & $1.82 \cdot 10^{-2}$ & $2.33 \cdot 10^{-2}$ \\
\hline \multicolumn{7}{|c|}{ Multipoint models } \\
\hline The number of points & 2 & 3 & 4 & 5 & 6 & 7 \\
\hline Annular layer in ${ }^{208} \mathrm{~Pb}$ reflector, $\mathrm{m}$ & $0.5 \div 1$ & $1 \div 2$ & $2 \div 3$ & $3 \div 4$ & $4 \div 5$ & $5 \div 6$ \\
\hline $\begin{array}{l}\text { Increase of the reactivity gain caused by thicker } \\
\text { reflector, } \$\end{array}$ & 3.01 & 2.29 & 1.21 & 0.69 & 0.37 & 0.20 \\
\hline Prompt neutron lifetime in the reflector layer, $s$ & $2.90 \cdot 10^{-5}$ & $2.36 \cdot 10^{-3}$ & $2.62 \cdot 10^{-2}$ & $7.25 \cdot 10^{-2}$ & $1.36 \cdot 10^{-1}$ & $2.16 \cdot 10^{-1}$ \\
\hline
\end{tabular}

a trivial task. A possible way of the solution is proposed in Appendix D.

\section{Physical Parameters of Neutron Reflector in Continuous Model of Neutron Kinetics}

Some relevant physical parameters of neutron reflector are considered here within the frames of spherically symmetrical continuous model of neutron kinetics and presented in Figure 2. It follows from Figure 2(a) that the reactivity gain thanks to the reflector $\rho_{R}(r)$ grows as the reflector becomes thicker and approaches a saturation level. Correspondingly, derivative of the reactivity gain decreases and vanishes. Derivative of the reactivity gain $d \rho_{R}(r) / d r$ defines the growth rate of contribution given by prompt neutrons to chain fission reaction as the reflector becomes thicker. So, it is natural that derivative of the reactivity gain decreases as the reflector thickness increases because contribution of neutrons coming back from more distant reflector layers into chain fission reaction in the reactor core vanishes.

It follows from the curves shown in Figure 2(b) that mean prompt neutron lifetime $\bar{\Lambda}_{\text {prt }}$ in the fast BREST-type reactor with ${ }^{208} \mathrm{~Pb}$ reflector as a whole becomes substantially longer (from $\sim 1 \mu \mathrm{sec}$ to $\sim 1 \mathrm{msec}$ ) as the reflector thickens from $0.5 \mathrm{~m}$ to $6 \mathrm{~m}$. Derivative of the mean prompt neutron lifetime has a weakly expressed peak at the reflector thickness of $\sim 3 \mathrm{~m}$. The latter means an important fact that elongation of mean prompt neutron lifetime becomes slower as thickness of the reflector exceeds $3 \mathrm{~m}$.

The following two terms of (10) are considered below, namely, $\Lambda_{R}(0)$, prompt neutron lifetime in the reflector layer of infinitesimal thickness adjacent to the reactor core, that is, practically without the reflector and $\Lambda_{\text {prt }}(0)$, prompt neutron lifetime in the reactor core only. The former is larger than the latter on the second summand. This is related with the following fact. Within the frames of one-point model, all prompt neutrons in the reactor core are characterized by a common generation time $\bar{\Lambda}_{C}$, averaged over the core volume, and it is assumed that $\Lambda_{\text {prt }}(0)=\bar{\Lambda}_{C}$. Lifetime of prompt neutrons of the reflector layer of even infinitesimal thickness, adjacent to the reactor core, is relatively longer because this lifetime is defined by neutrons that came up to the very edge

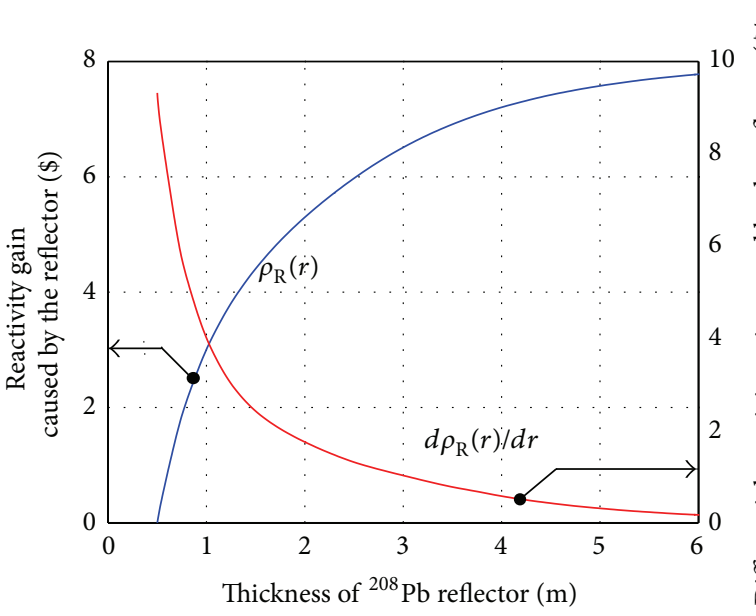

(a)

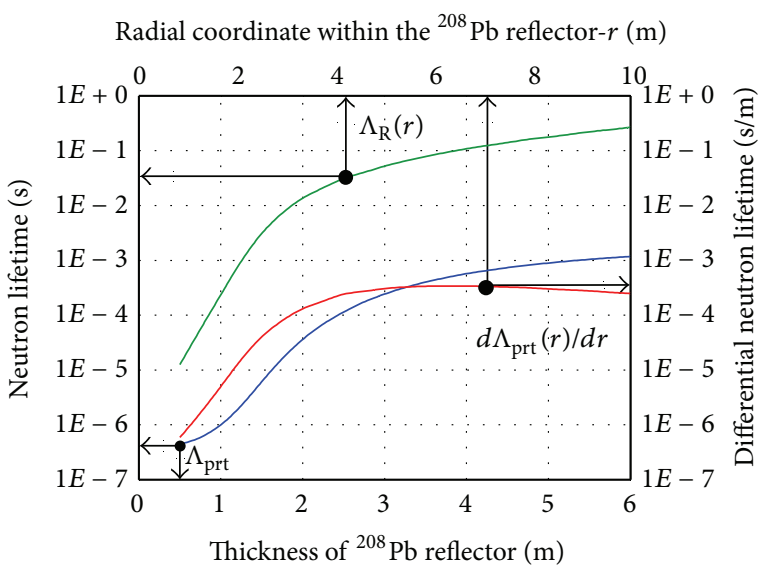

(b)

FIGURE 2: Physical parameters of the fast BREST-type reactor depending on thickness of neutron reflector made of ${ }^{208} \mathrm{~Pb}$.

of the reactor core and then came back to the reactor core. The explanation is confirmed by the calculated results presented in Figure 2(b). It can be seen that prompt neutron lifetime in the reflector layer of infinitesimal thickness $\Lambda_{R}(0)$ is longer 
than prompt neutron lifetime in the reactor without neutron reflector $\Lambda_{\text {prt }}(0)$ by above one order of magnitude.

As the reflector becomes thicker, difference between prompt neutron lifetime in the reflector $\Lambda_{R}(r)$ and mean prompt neutron lifetime in the reactor with neutron reflector $\bar{\Lambda}_{\text {prt }}$ increases. When the reflector thickness reaches $6 \mathrm{~m}$, the difference exceeds two orders of magnitude. It is noteworthy that lifetime of prompt neutrons coming back from 6-m-thick ${ }^{208} \mathrm{~Pb}$ reflector into the reactor core is equal to a rather long value, above $0.1 \mathrm{~s}$ (Figure 2(b)).

\section{Advancement of Continuous Model from $(R, T)$ Phase Space to $(\Lambda, T)$ Phase Space}

When physical parameters of physically thick neutron reflector were analyzed by using continuous neutron kinetics model, the following dependencies were calculated:

(i) Radial distribution (within the reflector thickness) of the contribution given by annular layer of unitary thickness into the reactivity gain $\rho_{R}(r)$ (Figure $2(\mathrm{a})$ ).

(ii) Radial distribution (within the reflector thickness) of prompt neutron lifetime in annular layer of unitary thickness $\Lambda_{R}(r)$ (Figure 2(b)).

This means that each annular layer of unitary thickness with inner radius $r$ is characterized by its own prompt neutron lifetime $\Lambda_{R}(r)$. Then, the contribution given by prompt neutrons with lifetime $\Lambda_{R}$ and longer into the reactivity gain may be considered as a certain generalized parameter of such a thick neutron reflector. This parameter defines capability of the reflector to generate prompt neutrons with different delay and can be calculated by using the following formulas:

$$
\rho_{R}\left(\Lambda_{R}, \Lambda_{\max }\right) \equiv \int_{r}^{r_{\text {out }}} \frac{d \rho_{R}\left(r^{\prime}\right)}{d r^{\prime}} \cdot d r^{\prime}=\int_{\Lambda_{R}}^{\Lambda_{\max }} \frac{d \rho_{R}}{d \Lambda_{R}^{\prime}} \cdot d \Lambda_{R}^{\prime}
$$

The contributions given by the neutron reflectors of different thickness into the reactivity gain as functions of neutron delay time in the reflector as long as $\Lambda_{R}$ and longer are presented in Figure 3. If the reflector is made of ${ }^{208} \mathrm{~Pb}$, then the contributions of neutrons with lifetimes of $1 \mathrm{msec}$ and longer into the reactivity gain can reach several dollars. For comparison, analogous dependency is shown in Figure 3 for the neutron reflector made of natural lead. In this case, the same reactivity effect caused by the reflector thickening and, as a consequence, by longer neutron lifetime remains insignificant even in the reflector with $2 \mathrm{~m}$ thickness.

Component of the reactivity gain-the second summand of the inverse-hour equation (9) — may be regarded as a useful characteristic parameter of the neutron reflector-converter:

$$
\rho_{R}^{*} \equiv \int_{r_{\text {in }}}^{r_{\text {out }}} \frac{d \rho_{R}\left(r^{\prime}\right) / d r^{\prime}}{1+T / \Lambda_{R}\left(r^{\prime}\right)} \cdot d r^{\prime} .
$$

One else informative function is an integrand in formula (12), whose radial distribution is shown in Figure 4.

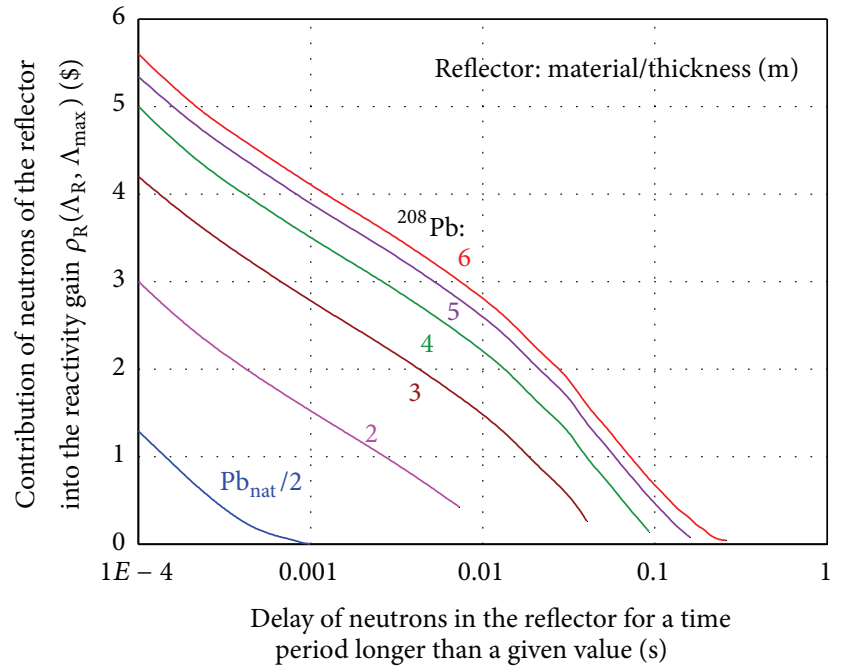

FIGURE 3: Radial distribution of the contribution given by neutrons of the reflector with lifetimes as long as $\Lambda_{R}$ and longer into the reactivity gain $\rho_{R}\left(\Lambda_{R}, \Lambda_{\max }\right)$. The reflector is made of ${ }^{208} \mathrm{~Pb}$ or natural lead, thickness-from $2 \mathrm{~m}$ to $6 \mathrm{~m}$.

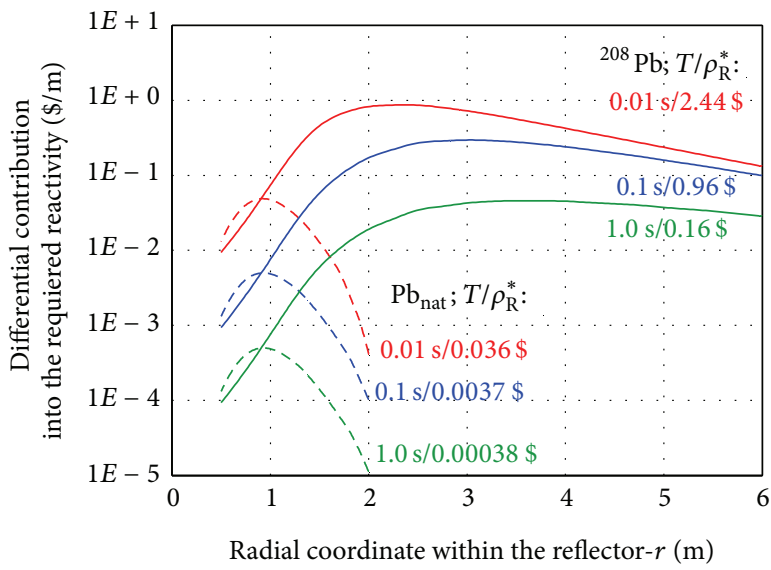

FIGURE 4: Differential contribution into the reactivity jump required to provide the power excursion with asymptotic time period $T$ (integrand of formula (12)).

To analyze the contributions of neutrons with various lifetimes in the reflector into the reactivity gain, it may be helpful to transform integration over radial coordinate into integration on neutron lifetime in the reflector $\Lambda_{R}$ :

$$
\begin{gathered}
\int_{r_{\text {in }}}^{r_{\text {out }}} \frac{d \rho_{R}\left(r^{\prime}\right) / d r^{\prime}}{1+T / \Lambda_{R}\left(r^{\prime}\right)} \cdot d r^{\prime}=\int_{\Lambda_{\min }}^{\Lambda_{\max }} \frac{d \rho_{R} / d \Lambda_{R}^{\prime}}{1+T / \Lambda_{R}^{\prime}} \cdot d \Lambda_{R}^{\prime} \\
\frac{d \rho_{R}}{d r}=\frac{d \rho_{R}}{d \Lambda_{R}} \cdot \frac{d \Lambda_{R}}{d r}
\end{gathered}
$$




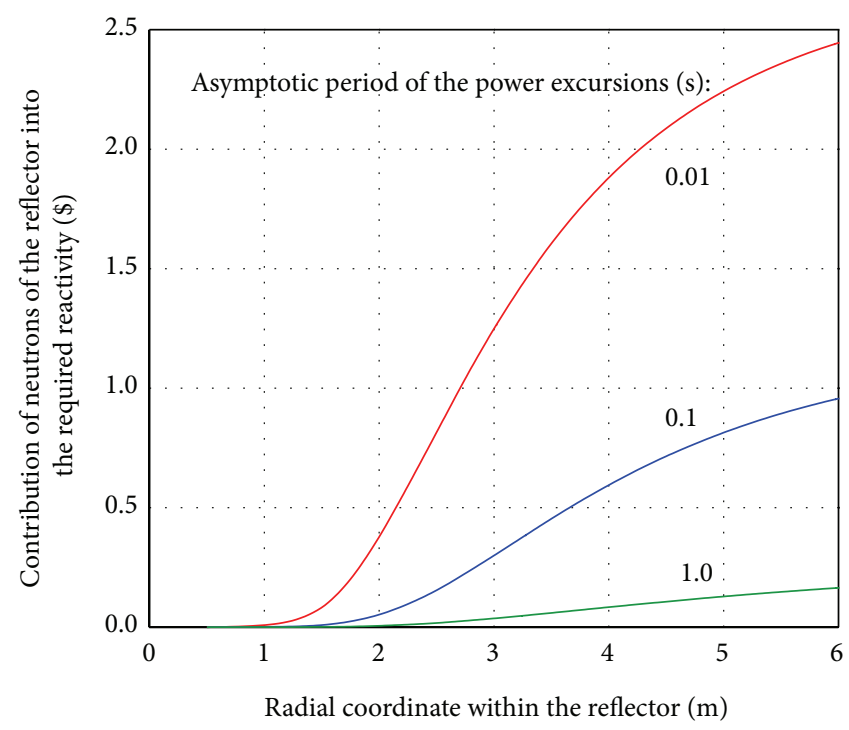

(a)

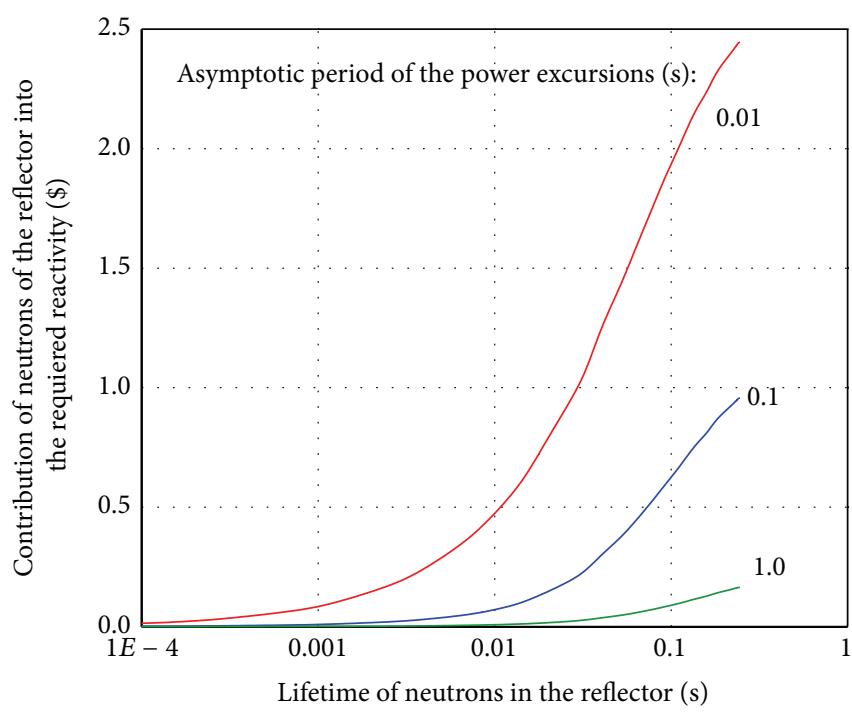

(b)

FIGURE 5: Component of the reactivity gain required to provide the power excursion with asymptotic time period $T$, which is defined by neutrons of the reflector layers adjacent to the reactor core (from $0.5 \mathrm{~m}$ to $r$, or from $\Lambda_{\text {min }}$ to $\Lambda_{R}$ ).

In this case, the inverse-hour equation may be re-written as follows:

$$
\begin{aligned}
\rho= & \frac{\bar{\Lambda}_{C}}{T}+\int_{\Lambda_{\min }}^{\Lambda_{\max }} \frac{\Lambda_{R}^{\prime} \cdot\left(d \rho_{R} / d \Lambda_{R}^{\prime}\right)}{\Lambda_{R}^{\prime}+T} \cdot d \Lambda_{R}^{\prime} \\
& +\sum_{i=1}^{6} \frac{\beta_{i}}{1+\lambda_{i} \cdot T} .
\end{aligned}
$$

It is interesting to note that the second and third summands in the inverse-hour equation have similar structures. Taking into account the fact that lifetime $\Lambda_{R}$ for major fraction of neutrons of the reflector is well below $0.1 \mathrm{~s}$, we can remove this value from denominator of the second summand in the inverse-hour equation (14) for the asymptotic time periods longer than $0.1 \mathrm{~s}$. As a result, the second summand becomes substantially simpler:

$$
\begin{aligned}
\int_{\Lambda_{\min }}^{\Lambda_{\max }} \frac{\Lambda_{R}^{\prime} \cdot\left(d \rho_{R} / d \Lambda_{R}^{\prime}\right)}{\Lambda_{R}^{\prime}+T} \cdot d \Lambda_{R}^{\prime} & =\frac{1}{T} \int_{\Lambda_{\min }}^{\Lambda_{\max }} \Lambda_{R}^{\prime} \cdot d \rho_{R} \\
& =\frac{1}{T} \int_{r_{\text {in }}}^{r_{\text {out }}} \Lambda_{R}^{\prime} \cdot d \rho_{R}\left(r^{\prime}\right) .
\end{aligned}
$$

Prompt neutron lifetime in the reflector is used here as a weighing function for the integration. Small values of the weighing function naturally decrease the calculated integrals. Since the decay constants $\lambda_{i}$ of delayed neutrons cover the range from $\sim 0.01$ to $\sim 3$ inverse seconds, the third summand in the inverse-hour equation (14) could not be simplified like the second summand.

Component of the reactivity gain required to provide the power excursion with asymptotic time period $T$, which is defined by neutrons of the reflector layers (from short-lived neutrons of the reflector layers adjacent to the reactor core and to relatively longer-lived neutrons in distant depth $r$, i.e., from $\Lambda_{\min }$ to current $\Lambda_{R}$ ), may be calculated as follows:

$$
\rho_{R}^{*}(r)=\int_{r_{\mathrm{in}}}^{r} \frac{d \rho_{R}\left(r^{\prime}\right) / d r^{\prime}}{1+T / \Lambda_{R}\left(r^{\prime}\right)} \cdot d r^{\prime}=\int_{\Lambda_{\min }}^{\Lambda} \frac{d \rho_{R} / d \Lambda_{R}^{\prime}}{1+T / \Lambda_{R}^{\prime}} \cdot d \Lambda_{R}^{\prime} .
$$

Radial and lifetime distributions of this component are presented in Figure 5.

As it follows from Figures 4 and 5, neutrons in distant layers of the reflector, that is, neutrons with relatively long lifetime $\Lambda_{R}$, give a dominant contribution into the reactivity gain.

If the asymptotic period of the power excursion is comparable or longer than time constant of fuel rods, then thermal energy can receive a long enough time period for its partial removal from fuel rods by coolant. Therefore, the process of fuel heating up slows down, and an opportunity arises for feedbacks on coolant density and temperature to actuate. Otherwise, if asymptotic period of the power excursion is substantially shorter than time constant of fuel rods, then fuel can be overheated and melted down. As the severest consequence, reactor can lose its ability of working. As for typical time constants of fuel rods, in experimental fast breeder reactor EBR-II (USA) fuel rods had time constants about $0.11 \mathrm{~s}$ [16]. If $1-\$$ positive reactivity is inserted into the fast BREST-type reactor with $0.5 \mathrm{~m}$ thick neutron reflector made of natural lead, then asymptotic period of the power excursion is considerably shorter $(0.014 \mathrm{~s})$ than time constant of its fuel rods. At the same time, if even 2 -\$ positive reactivity is inserted into the fast BREST-type reactor with $6 \mathrm{~m}$ thick neutron reflector made of ${ }^{208} \mathrm{~Pb}$, then asymptotic period of 
the power excursion becomes considerably longer ( $0.1 \mathrm{~s})$. That is why it is so important to develop fuel rods with quick heat transport to coolant. Then, reactor will be more stable and less vulnerable to the power excursions with short asymptotic periods caused by large reactivity jumps.

\section{Conclusions}

It is demonstrated that the use of radiogenic lead as a neutron reflector-converter makes it possible to slow down the chain fission reaction on prompt neutrons in the fast reactor and it can improve the nuclear safety of fast reactor.

(1) Multipoint model of neutron kinetics in nuclear reactors was applied to analyze time-dependent evolution of neutron population in fast reactor with physically thick neutron reflector made of weak neutron absorber (radiogenic lead with dominant content of $\left.{ }^{208} \mathrm{~Pb}\right)$

(2) Multipoint model of neutron kinetics was applied to investigate a possibility for substantial elongation of prompt neutron lifetime with correct accounting for time of neutron staying in physically thick, weak neutron-absorbing reflector.

(3) The paper demonstrates how multipoint discrete model of neutron kinetics can be transformed into continuous model of neutron kinetics in the reactor core surrounded by the neutron reflector.

(4) Numerical analyses of neutron kinetics were carried out with application of multipoint and continuous models and demonstrated that physically thick and weakly neutron-absorbing reflector is able to prolong prompt neutron lifetime in the fast BREST-type reactor by several orders of magnitude (roughly, from $0.5 \mu \mathrm{sec}$ to $1 \mathrm{msec}$ ). This effect can be interpreted as an appearance of one else group of delayed neutrons that increases their effective fraction and, thus, improves the reactor safety.

(5) Advanced design of fuel rods with quick heat transport from fuel to coolant can remarkably enhance the reactor resistance against the power excursions with short asymptotic periods caused by large reactivity jumps.

(6) If the asymptotic period of the power excursion caused by positive reactivity jump is comparable with thermal constant of fuel rods, then heat would have a time to flow down from fuel to coolant, its density would decrease and it would be able to impact remarkably on the power excursion process (see Appendix D). This allows us to consider the question: what positive reactivity jumps are permissible for the coolant-induced reactivity feedback could actuate?

\section{Appendices}

\section{A. Neutronic-Physical Properties and Advantages of Radiogenic Lead}

The term radiogenic lead is used here for designation of lead that is produced in radioactive decay chains of thorium and uranium isotopes. After a series of alpha and beta decays, ${ }^{232}$ Th transforms into stable lead isotope ${ }^{208} \mathrm{~Pb},{ }^{238} \mathrm{U}$ into stable lead isotope ${ }^{206} \mathrm{~Pb}$, and ${ }^{235} \mathrm{U}$ into stable lead isotope ${ }^{207} \mathrm{~Pb}$. Therefore, uranium ores contain radiogenic lead consisting mainly of ${ }^{206} \mathrm{~Pb}$, while thorium and mixed thorium-uranium ores contain radiogenic lead consisting mainly of ${ }^{208} \mathrm{~Pb}$. Sometimes, the presence of natural lead in uranium and thorium ores can change isotope composition of radiogenic lead. Anyway, isotope composition of radiogenic lead depends on the elemental composition of the ores from which this lead is extracted.

Radiogenic lead consisting mainly of stable lead isotope ${ }^{208} \mathrm{~Pb}$ can offer unique advantages, which follow from unique nuclear physics properties of ${ }^{208} \mathrm{~Pb}$. This lead isotope is a double-magic nuclide with completely closed neutron and proton shells. The excitation levels of ${ }^{208} \mathrm{~Pb}$ nuclei (Figure 6) are characterized by a high value of energy (the energy of the first level being $2.61 \mathrm{MeV}$ ), while the first excitation levels of other lead isotopes have lower energy range $(0.57$ to $0.90 \mathrm{MeV})$. This results in the fact that the threshold in energy dependence of ${ }^{208} \mathrm{~Pb}$ inelastic scattering cross-section is at much higher energy than those of other lead isotopes [17] (Figure 7).

The unique nuclear properties of ${ }^{208} \mathrm{~Pb}$ can be used to improve parameters of chain fission reaction in a nuclear reactor. First, since energy threshold of inelastic neutron scattering by ${ }^{208} \mathrm{~Pb}(\sim 2.61 \mathrm{MeV})$ is substantially higher than that by natural lead $(\sim 0.8 \mathrm{MeV}),{ }^{208} \mathrm{~Pb}$ can soften neutron spectrum in the high-energy range to a remarkably lower degree. Second, neutron radiative capture cross section of ${ }^{208} \mathrm{~Pb}$ in thermal point $(\sim 0.23 \mathrm{mb})$ is smaller by two orders of magnitude than that of natural lead $(\sim 174 \mathrm{mb})$ and even smaller than that of reactor-grade graphite $(\sim 3.9 \mathrm{mb})$. These differences remain large within sufficiently wide energy range (from thermal energy to some tens of kilo-electron-volts). Energy dependence of neutron absorption cross sections is presented in Figure 8 for natural lead, stable lead isotopes, graphite, and deuterium [17].

If radiogenic lead consists mainly of ${ }^{208} \mathrm{~Pb}\left(\sim 90 \%{ }^{208} \mathrm{~Pb}\right.$ plus $9 \%{ }^{206} \mathrm{~Pb}$ and $\sim 1 \%{ }^{204} \mathrm{~Pb}+{ }^{206} \mathrm{~Pb}$ ), then such a lead absorbs neutrons is as weak as graphite within the energy range from $0.01 \mathrm{eV}$ to $1 \mathrm{keV}$. Such isotope compositions of radiogenic lead can be found in thorium and in mixed thorium-uranium ores.

Thus, on one hand, ${ }^{208} \mathrm{~Pb}$, being heavy nuclide, is a relatively weak neutron moderator both in elastic scattering reactions within full neutron energy range of nuclear reactors because of heavy atomic mass and in inelastic scattering reactions with fast neutrons because of high energy threshold of these reactions. On the other hand, ${ }^{208} \mathrm{~Pb}$ is an extremely weak neutron absorber within wide enough energy range. 

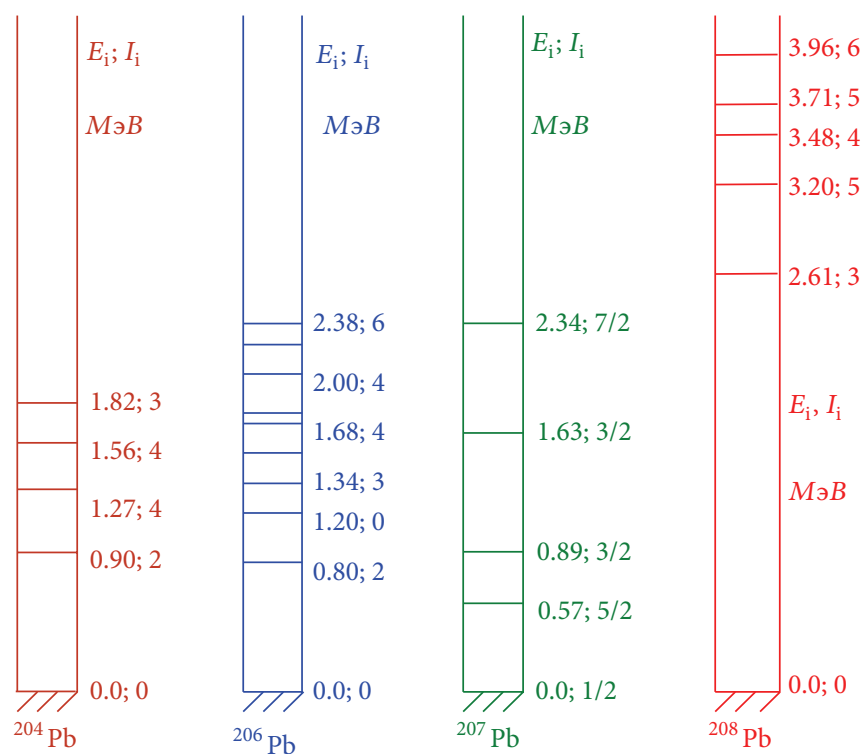

FIgURE 6: The excitation levels of lead nuclei.

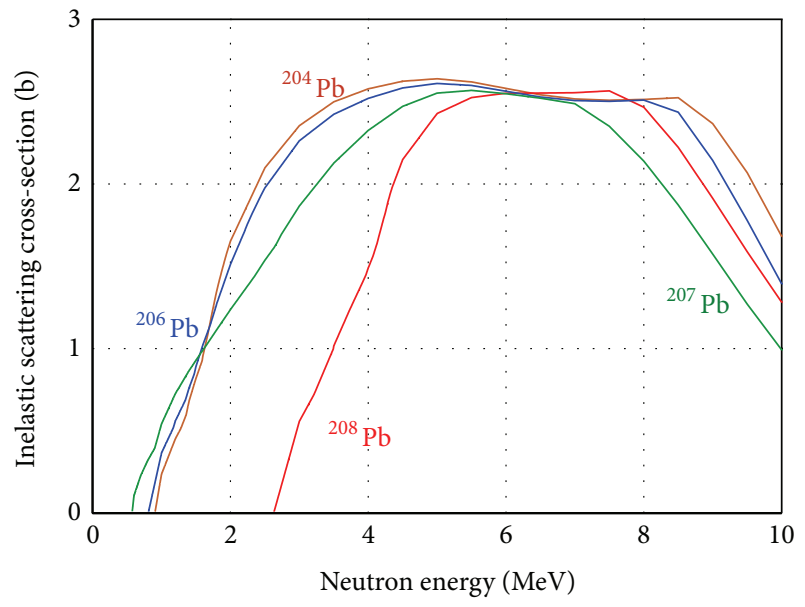

Figure 7: Inelastic scattering cross-section of lead isotopes as a function of neutron energy.

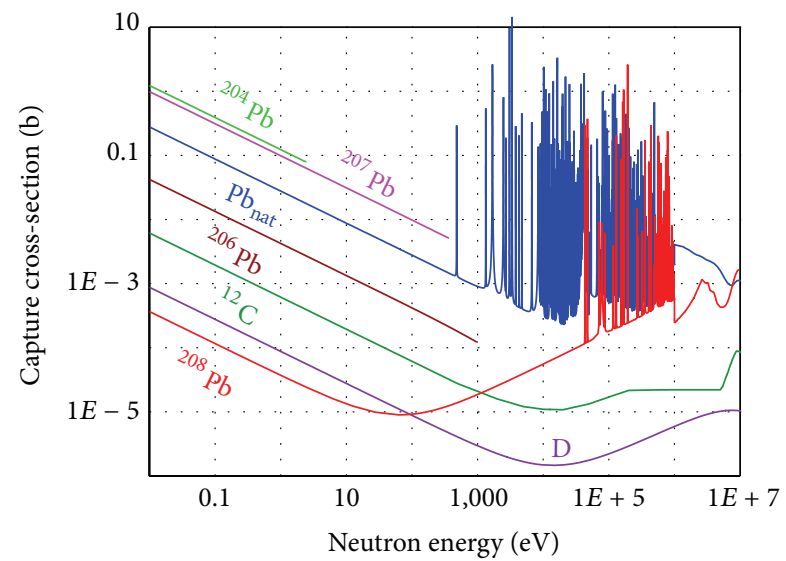

FIGURE 8: Capture cross sections of various nuclides as a function of neutron energy (JENDL-4.0).
Some nuclear characteristics of light neutron moderators (hydrogen, deuterium, beryllium, graphite, and oxygen) and heavy materials (natural lead and lead isotope ${ }^{208} \mathrm{~Pb}$ ) are presented in Table 2 [17].

One can see that elastic cross sections of natural lead and ${ }^{208} \mathrm{~Pb}$ do not differ significantly from the others nuclides, being between the corresponding values for hydrogen and other light nuclides. Neutron slowing-down from $0.1 \mathrm{MeV}$ to $0.5 \mathrm{eV}$ requires from 12 to 102 elastic collisions with light nuclides, while the same neutron slowing-down requires 1270 elastic collisions with natural lead or ${ }^{208} \mathrm{~Pb}$. The reason is the high atomic mass of lead in comparison with the other light nuclides. From this point of view neither natural lead nor ${ }^{208} \mathrm{~Pb}$ are effective neutron moderators.

Since ${ }^{208} \mathrm{~Pb}$ is a double magic nuclide with closed proton and neutron shells, radiative capture cross-section at thermal energy and resonance integral of ${ }^{208} \mathrm{~Pb}$ is much smaller than the corresponding values of lighter nuclides. Therefore, it can be expected that even with multiple scattering of neutrons on ${ }^{208} \mathrm{~Pb}$ during the process of their slowing-down, they will be slowed down with a high probability and will create high flux of slowed down neutrons.

So, thanks to very small neutron capture cross section, the moderating ratio (see Table 3) [17-19], that is, the average logarithmic energy loss times scattering cross section divided by thermal absorption cross section, of ${ }^{208} \mathrm{~Pb}$ is much higher than that for light moderators. This means that ${ }^{208} \mathrm{~Pb}$ could be a more effective moderator than such well-known light moderators as light water, beryllium oxide, and graphite.

It is noteworthy that mean lifetime of thermal neutrons in ${ }^{208} \mathrm{~Pb}$ is very large $(\sim 0.6 \mathrm{~s})$. This effect could be used to essentially improve the safety of the fast reactor by slowing down progression of chain fission reaction on prompt neutrons [6]. This assumes the greater significance under accidental conditions with destruction of the reactor core, 
TABLE 2: Neutron-physical characteristics of some materials.

\begin{tabular}{lcccc}
\hline Nuclide & $\sigma_{\mathrm{el}}^{10 \mathrm{eV}}(\mathrm{b})$ & $\begin{array}{c}\text { Number of collisions } \\
(0.1 \mathrm{MeV} \rightarrow 0.5 \mathrm{eV})\end{array}$ & $\sigma_{(n, \gamma)}^{\text {th }}(\mathrm{mb})$ & $R I_{n, \gamma}+1 / V(\mathrm{mb})$ \\
\hline${ }^{1} \mathrm{H}$ & 30.1 & 12 & 332 & 0.55 \\
${ }^{2} \mathrm{D}$ & 4.2 & 17 & 8.5 & 0.25 \\
${ }^{9} \mathrm{Be}$ & 6.5 & 59 & 3.9 & 3.8 \\
${ }^{12} \mathrm{C}$ & 4.9 & 77 & 0.19 & 1.8 \\
${ }^{16} \mathrm{O}$ & 4.0 & 102 & 174 & 0.16 \\
$\mathrm{~Pb}_{\text {nat }}$ & 11.3 & 1269 & 0.23 & 0.78 \\
${ }^{208} \mathrm{~Pb}$ & 11.5 & 1274 & 0.78 \\
\hline
\end{tabular}

TABle 3: Properties of neutron moderators at $20^{\circ} \mathrm{C}$.

\begin{tabular}{lccccc}
\hline Moderator & $\begin{array}{c}\text { Average logarithmic } \\
\text { energy loss } \xi\end{array}$ & $\begin{array}{c}\text { Moderating } \\
\text { ratio } \\
\xi \Sigma / \Sigma_{a}\end{array}$ & $\begin{array}{c}\text { Neutron age } \tau \\
(0.1 \mathrm{MeV} \rightarrow 0.5 \mathrm{eV}) \\
\left(\mathrm{cm}^{2}\right)\end{array}$ & $\begin{array}{c}\text { Diffusion length } \\
L(\mathrm{~cm})\end{array}$ & $\begin{array}{c}\text { Mean lifetime } T_{\text {th }} \text { of } \\
\text { thermal neutrons } \\
(\mathrm{ms})\end{array}$ \\
\hline $\mathrm{H}_{2} \mathrm{O}$ & 0.95 & 70 & 6 & 3 & 0.2 \\
$\mathrm{D}_{2} \mathrm{O}$ & 0.57 & 4590 & 58 & 66 & 147 \\
$\mathrm{BeO}$ & 0.17 & 247 & 160 & 37 & 130 \\
${ }^{12} \mathrm{C}$ & 0.16 & 242 & 3033 & 56 & 13 \\
$\mathrm{~Pb}_{\text {nat }}$ & 0.00962 & 0.6 & 2979 & 341 & 0.8 \\
${ }^{208} \mathrm{~Pb}$ & 0.00958 & 477 & & & 598 \\
\hline
\end{tabular}

when the energy yield is defined by lifetime of prompt neutrons because other kinetic parameters either out of any control (the inserted positive reactivity) or cannot go out of the very limited range (Doppler coefficient, effective fraction of delayed neutrons, the reactor power).

It should be noted that ${ }^{208} \mathrm{~Pb}$ is not the only of the kind nuclide in Mendeleev's periodic system whose neutronphysical properties are very specifics. For example, nuclide ${ }^{88} \mathrm{Sr}$ has a very small neutron capture as well, inelastic cross section even smaller than that for ${ }^{208} \mathrm{~Pb}$ nuclide, and values of nuclear moderating capabilities $\xi * \sigma_{s}$ are close for ${ }^{88} \mathrm{Sr}$ and ${ }^{208} \mathrm{~Pb}$ [17].

Table 4 summarizes relevant neutron-physical characteristics of some nuclear materials, including radiogenic lead. It can be seen that natural lead is able to slow down only $30.4 \%$ of fast neutrons $(0.1 \mathrm{MeV})$ into epi-cadmium range $(0.5 \mathrm{eV})$, while the remaining fraction $(69.6 \%)$ is absorbed by natural lead in the slowing-down process. On the contrary, almost all fast neutrons (99.3\%) can be slowed down by ${ }^{208} \mathrm{~Pb}$ into epithermal range.

It is worthy to note that, in both cases, that is, during the slowing-down process of fast neutrons in natural lead and in ${ }^{208} \mathrm{~Pb}$, mean distance of neutron transport and mean slowing-down time are approximately the same $(\sim 134 \mathrm{~cm}$ and $0.56 \mathrm{~ms}$ ). As for thermal neutrons, mean distances of neutron diffusion until absorption are quite different for the two lead types $\left(30 \mathrm{~cm}\right.$ in natural lead and $835 \mathrm{~cm}$ in $\left.{ }^{208} \mathrm{~Pb}\right)$.

This means that, first, very small fraction of neutrons that were slowed down in natural lead reflector can come back into the reactor core. On the contrary, ${ }^{208} \mathrm{~Pb}$ reflector gives them such a possibility. Second, mean lifetime of thermal neutrons in the infinite ${ }^{208} \mathrm{~Pb}$ environment $(0.6 \mathrm{~s})$ is longer by three orders of magnitude than that in natural lead $(0.8 \mathrm{~ms})$. So, main process in ${ }^{208} \mathrm{~Pb}$ reflector is a neutron slowing down, not neutron absorption, and these slow neutrons, after a diffusion (and time delay), have a probability to come back into the reactor core and sustain the chain fission reaction. Mean lifetimes of slow neutrons and, especially, thermal neutrons are substantially longer than those for fast and slowing-down neutrons. This constitutes a potential for significant extension of mean prompt neutron lifetime in the chain fission reaction.

It is noteworthy that neutron-physical parameters of radiogenic lead extracted from thorium and thoriumuranium ore deposits in Brazil, Australia, the United States, and the Ukraine are inferior to those of ${ }^{208} \mathrm{~Pb}$, but they are substantially better than those of natural lead (radiogenic lead compositions from different deposits are presented in the appendix). For comparison, Table 4 presents relevant data for heavy water and graphite. Some neutron-physical parameters of these materials are superior to those of ${ }^{208} \mathrm{~Pb}$. However, the use of heavy water or graphite as a neutron reflector in a fast reactor is a doubtful option, at least, because such a neutron reflector can substantially soften neutron spectrum in the reactor core with all negative consequences.

\section{B. Natural Resources of Radiogenic Lead}

In nature there are two types of elemental lead with substantially different contents of four stable lead isotopes $\left({ }^{204} \mathrm{~Pb}\right.$, ${ }^{206} \mathrm{~Pb},{ }^{207} \mathrm{~Pb}$, and ${ }^{208} \mathrm{~Pb}$ ). The first type is a natural, or common, lead with a constant isotopic composition $(1.4 \%$ 
TABLE 4: Neutron-physical characteristics of different materials.

\begin{tabular}{|c|c|c|c|c|c|}
\hline \multirow{2}{*}{ Material } & \multirow{2}{*}{$\begin{array}{c}\text { Slowing-down } \\
\text { probability } \\
(0.1 \mathrm{MeV} \rightarrow 0.5 \mathrm{eV})\end{array}$} & \multirow{2}{*}{$\sqrt{\overline{r_{E}^{2}}}=\sqrt{6 \tau}(\mathrm{cm})$} & \multirow{2}{*}{$\sqrt{\overline{r_{\mathrm{th}}^{2}}}=\sqrt{6} L(\mathrm{~cm})$} & \multicolumn{2}{|c|}{ Neutron lifetime (ms) } \\
\hline & & & & Slowing down & Thermal \\
\hline $\mathrm{Pb}_{\text {nat }}$ & 0.304 & 134 & 30 & 0.56 & 0.8 \\
\hline${ }^{208} \mathrm{~Pb}$ & 0.993 & 134 & 835 & 0.56 & 598 \\
\hline $\mathrm{D}_{2} \mathrm{O}$ & 0.999 & 19 & 360 & 0.01 & 130 \\
\hline Graphite & 0.998 & 31 & 138 & 0.03 & 13 \\
\hline \multicolumn{6}{|c|}{ Radiogenic $\mathrm{Pb}$ from different deposits } \\
\hline Brazil & 0.930 & 134 & 186 & 0.56 & 29 \\
\hline Australia & 0.914 & 134 & 142 & 0.56 & 17 \\
\hline The USA & 0.885 & 134 & 129 & 0.56 & 14 \\
\hline Ukraine & 0.915 & 134 & 145 & 0.56 & 18 \\
\hline
\end{tabular}

${ }^{204} \mathrm{~Pb}, 24.1 \%{ }^{206} \mathrm{~Pb}, 22.1 \%{ }^{207} \mathrm{~Pb}$, and $\left.52.4 \%{ }^{208} \mathrm{~Pb}\right)$. The second type is a so-called radiogenic lead with very variable isotopic composition. Radiogenic lead is a final product of radioactive decay chains in uranium and thorium ores. That is why isotopic compositions of radiogenic lead are defined by the ore age and by elemental compositions of mixed thorium-uranium ores sometimes with admixture of natural (common) lead as an impurity. The isotopes ${ }^{208} \mathrm{~Pb},{ }^{206} \mathrm{~Pb}$, and ${ }^{207} \mathrm{~Pb}$ are the final products of the radioactive decay chains starting from ${ }^{232} \mathrm{Th},{ }^{238} \mathrm{U}$, and ${ }^{235} \mathrm{U}$, respectively:

$$
\begin{gathered}
{ }^{232} \mathrm{Th} \longrightarrow 6 \alpha+4 \beta\left(14.6 \cdot 10^{9} \text { years }\right) \longrightarrow{ }^{208} \mathrm{~Pb}, \\
{ }^{238} \mathrm{U} \longrightarrow 8 \alpha+6 \beta\left(4.6 \cdot 10^{9} \text { years }\right) \longrightarrow{ }^{206} \mathrm{~Pb}, \\
{ }^{235} \mathrm{U} \longrightarrow 7 \alpha+4 \beta\left(0.7 \cdot 10^{9} \text { years }\right) \longrightarrow{ }^{207} \mathrm{~Pb} .
\end{gathered}
$$

Therefore, radiogenic lead with large abundance of ${ }^{208} \mathrm{~Pb}$ could be extracted from natural thorium and thoriumUranium ores [20-24] without any isotope separation procedures.

It should be noted that neutron capture cross-sections of ${ }^{206} \mathrm{~Pb}$, although larger than those of ${ }^{208} \mathrm{~Pb}$, are significantly smaller than those of ${ }^{207} \mathrm{~Pb}$ and ${ }^{204} \mathrm{~Pb}$. Thus, at first glance it appears that the ores containing $\sim 93 \%{ }^{208} \mathrm{~Pb}$ and $6 \%$ ${ }^{206} \mathrm{~Pb}$ (Table 5) could provide the necessary composition of radiogenic lead. However, the first estimations showed that the content of only $1 \%{ }^{204} \mathrm{~Pb}$ and ${ }^{207} \mathrm{~Pb}$ (these isotopes have high values of capture cross-sections) in radiogenic lead could significantly weaken the advantages of radiogenic lead in thermal nuclear reactors.

So, radiogenic lead can be taken as a byproduct from the process of uranium and thorium ores mining. Until now, extraction of uranium or thorium from minerals had been followed by throwing radiogenic lead into tail repositories. If further studies will reveal the perspective for application of radiogenic lead in nuclear power industry, then a necessity arises to arrange byextraction of radiogenic lead from thorium and uranium deposits or tails. Evidently, the scope of the ores mining and processing is defined by the demands for uranium and thorium.

However, the demands of nuclear power industry for thorium are quite small now and will remain so in the near future. Nevertheless, there is one important factor that can produce a substantial effect on the scope of thorium and mixed thorium-uranium ore mining. In the majority of cases, uranium and thorium ores belong to the complexore category, that is, they contain minor amounts of many valuable metals (rare-earth elements, gold and so on).

The paper by Sinev [25] has demonstrated that the presence of useful accompanying elements (some elements of cerium group, in particular) in uranium and thorium ores might be a factor of high significance for making cheaper the process of natural uranium and thorium production. Byextraction of some valuable elements from uranium ores can drop the smaller limit (industrial minimum) of uranium content in ores to $0.01 \%$ to $0.03 \%$ under application of the existing technologies for natural uranium extraction. Radiogenic lead can be recovered from the available tail repositories or as a byproduct of the processes applied for extraction of the accompanying valuable metals from uranium and thorium ores [26].

\section{Derivation of Balance Relationship}

The balance relationship can be used to evaluate mean prompt neutron lifetime in a multizone fast reactor with the reactor core surrounded by neutron reflector-converter to slow down the chain fission reaction under accidental power excursions. As is expected, the balance relationship allows us to determine spatial (zone-wise) contributions into mean prompt neutron lifetime.

To make the derivation as clear as possible, the simplest spherical two-zone model (Figure 9) of nuclear reactor is considered below. The model includes the reactor core surrounded by the neutron reflector. It is assumed the neutron reflector is made of heavy material with weak neutron absorption (lead isotope ${ }^{208} \mathrm{~Pb}$, e.g.).

As is well-known [1], mean prompt neutron lifetime is a bilinear fractional functional of space-energy distributions of neutron flux $n(r, E)$ and its adjoint function $n^{+}(r, E)$ :

$$
\bar{\Lambda}_{\mathrm{prt}} \equiv \frac{\left\langle n, n^{+}\right\rangle}{\left\langle v_{f} \Sigma_{f} \cdot v \cdot n \cdot n^{+}\right\rangle},
$$


TABle 5: Main deposits of uranium, thorium, and mixed uranium-thorium ores. Elemental compositions of minerals and isotope compositions of radiogenic lead.

\begin{tabular}{lccc}
\hline Deposit & $\mathrm{U} / \mathrm{Th} / \mathrm{Pb}($ wt. $\%)$ & ${ }^{204} \mathrm{~Pb} /{ }^{206} \mathrm{~Pb} /{ }^{207} \mathrm{~Pb} /{ }^{208} \mathrm{~Pb}($ at. $\%)$ & $\mathrm{Age}\left(\times 10^{9} \mathrm{yr}\right)$ \\
\hline Monazite, Guarapari, Brazil & $1.3 / 59.3 / 1.5$ & $0.005 / 6.03 / 0.46 / 93.5$ & 0.52 to 0.55 \\
Monazite, Manitoba, Canada & $0.3 / 15.6 / 1.5$ & $0.01 / 10.2 / 1.86 / 87.9$ & 1.83 to 3.18 \\
Monazite, Mt. Isa Mine, Australia & $0.0 / 5.73 / 0.3$ & $0.038 / 5.44 / 0.97 / 93.6$ & 1.00 to 1.19 \\
Monazite, Las Vegas, Nevada & $0.1 / 9.39 / 0.4$ & $0.025 / 9.07 / 1.13 / 89.8$ & 0.77 to 1.73 \\
Uraninite, Singar Mine, India & $64.3 / 8.1 / 8.9$ & $-/ 89.4 / 6.44 / 4.18$ & 0.885 \\
Monazite, South Bug, Ukraine & $0.2 / 8.72 / 0.9$ & $0.01 / 6.04 / 0.94 / 93.0$ & 1.8 to 2.0 \\
\hline
\end{tabular}

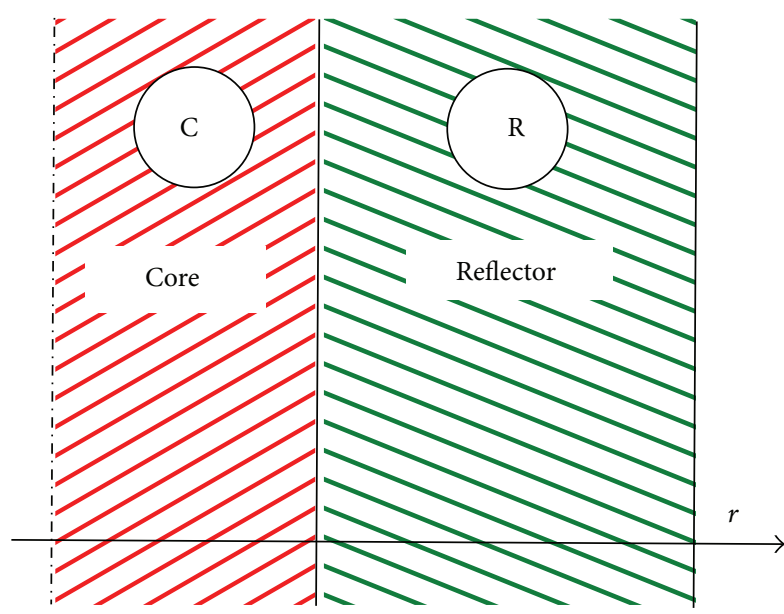

FIgURE 9: Two-zone model of nuclear reactor.

where the brackets $\langle\cdot\rangle$ designate integration on space and energy intervals.

Space-energy distributions of neutron flux and its adjoint function are defined by the following operator equations:

$$
\begin{gathered}
\widehat{L} n=\frac{1}{K_{\mathrm{ef}}} \cdot \widehat{\mathrm{Q}} n ; \\
\widehat{L}^{+} n^{+}=\frac{1}{K_{\mathrm{ef}}} \cdot \widehat{\mathrm{Q}}^{+} n^{+},
\end{gathered}
$$

where operator $\widehat{L}$ describes neutron transport, absorption, and scattering and operator $\widehat{Q}$ describes neutron multiplication by fission reactions.

As applied to the two-zone model under consideration here (the reactor core surrounded by the neutron reflector), the following designations will be used below: the bracket $\langle\cdot\rangle_{C}$ means integration on full volume of the reactor core, the bracket $\langle\cdot\rangle_{R}$ means integration on full volume of the neutron reflector, and the bracket $\langle\cdot\rangle_{C+R}$ means integration on full volume of the reactor (core plus reflector).

Two problems must be solved to evaluate prompt neutron lifetimes in the reactor zones. The first problem consists in solving (C.3) and (C.4) to determine space-energy distributions of neutron flux $n_{C+R}(r, E)$ and its adjoint function $n_{\mathrm{C}+R}^{+}(r, E)$ in two-zone reactor:

$$
\begin{aligned}
& \widehat{L}_{C+R} n_{C+R}=\frac{1}{K_{\mathrm{ef}}} \cdot \widehat{\mathrm{Q}} n_{\mathrm{C}+R} \\
& \widehat{L}_{\mathrm{C}+R}^{+} n_{\mathrm{C}+R}^{+}=\frac{1}{K_{\mathrm{ef}}} \cdot \widehat{\mathrm{Q}}^{+} n_{\mathrm{C}+R}^{+} .
\end{aligned}
$$

The second problem is to determine space-energy distribution of neutron flux $n_{C}(r, E)$ in the reactor core only, that is, without the neutron reflector, by solving (C.5):

$$
\widehat{L}_{C} n_{C}=\frac{1}{K_{\text {ef }}} \cdot \widehat{Q} n_{C+R}
$$

Solution of (C.5) for the bare core $n_{C}(r, E)$ characterizes those neutrons which, being generated in the reactor core, do not escape the core. Solution of (C.3) for the reflected core $n_{C+R}(r, E)$ characterizes those neutrons, which, being generated in the reactor core, can escape or stay in the core, thus populating both reactor zones. Their difference $\Delta n_{C+R}=\left(n_{C+R}-n_{C}\right)$ characterizes those neutrons which, being generated in the reactor core, escaped the core, went into the neutron reflector and then, in the migration process, populated both reactor zones (Figure 10).

Some fraction of those neutrons which, being generated in the reactor core, escaped the core, went into the neutron reflector and then came back into the reactor core, could contribute to time-dependent evolution of the chain fission reaction. Just these neutrons can be characterized by a relatively longer lifetime due to the lengthy processes of their diffusion, scattering and slowing down in the weakly absorbing neutron reflector, due to the processes of their coming back to the reactor core and contributing into the chain fission reaction.

Equation (C.1) that determines mean prompt neutron lifetime can be rewritten for the two-zone model as follows:

$$
\bar{\Lambda}_{\mathrm{prt}} \equiv \frac{\left\langle n_{\mathrm{C}+R}, n_{\mathrm{C}+R}^{+}\right\rangle_{\mathrm{C}+R}}{\left\langle v_{f} \Sigma_{f} \cdot v \cdot n_{C+R} \cdot n_{C+R}^{+}\right\rangle_{C+R}} .
$$

The space-energy distribution of neutron flux $n_{C+R}$ can be replaced by $n_{C+R}=n_{C}+\Delta n_{C+R}$. After some simple algebraic 


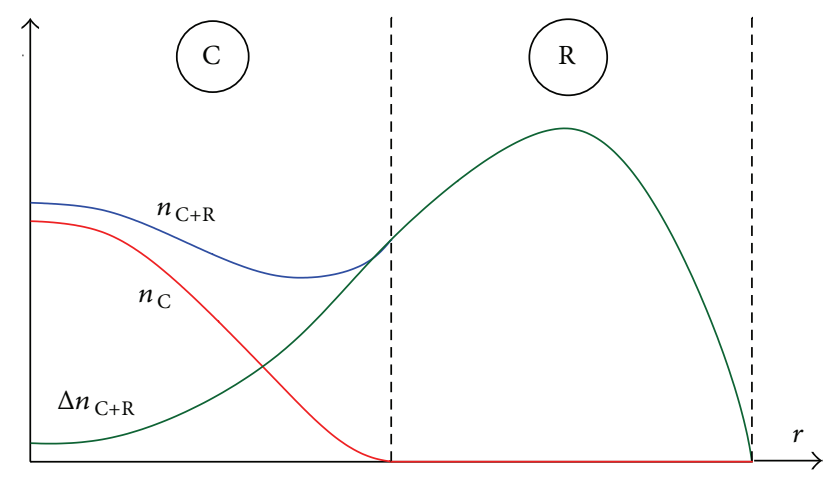

FIGURE 10: Model spatial distributions of neutron flux in the reflected core $n_{C+R}(r)$, in the bare core $n_{C}(r)$, and their difference $\Delta n_{C+R}(r)$.

operations, the following expression for mean prompt neutron lifetime can be obtained:

$$
\begin{aligned}
\bar{\Lambda}_{\mathrm{prt}}= & \frac{\left\langle\left(n_{C}+\Delta n_{C+R}\right), n_{C+R}^{+}\right\rangle_{C+R}}{\left\langle v_{f} \Sigma_{f} \cdot v \cdot n_{C+R} \cdot n_{C+R}^{+}\right\rangle_{C+R}} \\
= & \frac{\left\langle n_{C}, n_{C+R}^{+}\right\rangle_{C+R}}{\left\langle v_{f} \Sigma_{f} \cdot v \cdot n_{C+R} \cdot n_{C+R}^{+}\right\rangle_{C+R}} \\
& +\frac{\left\langle\Delta n_{C+R}, n_{C+R}^{+}\right\rangle_{C+R}}{\left\langle v_{f} \Sigma_{f} \cdot v \cdot n_{C+R} \cdot n_{C+R}^{+}\right\rangle_{C+R}} .
\end{aligned}
$$

If the first summand of formula (C.7) is divided by and multiplied on $\left\langle v_{f} \Sigma_{f} \cdot v \cdot n_{c} \cdot n_{C+R}^{+}\right\rangle_{C}$, the summand transforms into the following form:

$$
\begin{gathered}
\frac{\left\langle n_{C}, n_{C+R}^{+}\right\rangle_{C+R}}{\left\langle v_{f} \Sigma_{f} \cdot v \cdot n_{C+R} \cdot n_{C+R}^{+}\right\rangle_{C+R}} \\
=\frac{\left\langle n_{C}, n_{C+R}^{+}\right\rangle_{C}}{\left\langle v_{f} \Sigma_{f} \cdot v \cdot n_{C} \cdot n_{C+R}^{+}\right\rangle_{C}} \\
\cdot \frac{\left\langle v_{f} \Sigma_{f} \cdot v \cdot n_{C} \cdot n_{C+R}^{+}\right\rangle_{C+R}}{\left\langle v_{f} \Sigma_{f} \cdot v \cdot n_{C+R} \cdot n_{C+R}^{+}\right\rangle_{C+R}} .
\end{gathered}
$$

Note the integration range of the first fraction is shortened to the reactor core while the integration range of the second fraction is widened up to the full reactor volume. This may be done because, in both cases, the integration is performed with weight of neutron flux in the bare core $n_{C}(r, E)$ that is equal to zero in the neutron reflector (Figure 10). As it can be seen, the first fraction is a mean prompt neutron lifetime in the reactor core, that is, mean lifetime of those neutrons, which did not escape the reactor core:

$$
\bar{\Lambda}_{C} \equiv \frac{\left\langle n_{C}, n_{C+R}^{+}\right\rangle_{C}}{\left\langle v_{f} \Sigma_{f} \cdot v \cdot n_{C} \cdot n_{C+R}^{+}\right\rangle_{C}}
$$

The second fraction is a ratio between the value of fission neutrons produced by those neutrons, which did not escape the reactor core, and the value of fission neutrons produced by all neutrons including those neutrons, which escaped the reactor core, went into the neutron reflector then came back into the reactor core and contributed into the chain fission reaction. As $n_{C}=\left(n_{C+R}-\Delta n_{C+R}\right)$, then, after some simple algebraic operations, the following formula can be obtained:

$$
\begin{gathered}
\frac{\left\langle v_{f} \Sigma_{f} \cdot v \cdot\left[n_{C+R}-\Delta n_{C+R}\right] \cdot n_{C+R}^{+}\right\rangle_{C+R}}{\left\langle v_{f} \Sigma_{f} \cdot v \cdot n_{C+R} \cdot n_{C+R}^{+}\right\rangle_{C+R}} \\
=1-\frac{\left\langle v_{f} \Sigma_{f} \cdot v \cdot \Delta n_{C+R} \cdot n_{C+R}^{+}\right\rangle_{C+R}}{\left\langle v_{f} \Sigma_{f} \cdot v \cdot n_{C+R} \cdot n_{C+R}^{+}\right\rangle_{C+R}} .
\end{gathered}
$$

The second term in the right part is a ratio between the value of fission neutrons produced by those neutrons, which came back from the neutron reflector into the reactor core, and the value of fission neutrons produced by all neutrons. This is, in essence, a contribution into total reactivity $\rho_{R}$ from those neutrons, which came back from the neutron reflector:

$$
\rho_{R}=\frac{\left\langle v_{f} \Sigma_{f} \cdot v \cdot \Delta n_{C+R} \cdot n_{C+R}^{+}\right\rangle_{C+R}}{\left\langle v_{f} \Sigma_{f} \cdot v \cdot n_{C+R} \cdot n_{C+R}^{+}\right\rangle_{C+R}} .
$$

So, the first summand of formula (C.7) can be written in the following form:

$$
\frac{\left\langle n_{C}, n_{C+R}^{+}\right\rangle_{C+R}}{\left\langle v_{f} \Sigma_{f} \cdot v \cdot n_{C+R} \cdot n_{C+R}^{+}\right\rangle_{C+R}}=\bar{\Lambda}_{C} \cdot\left(1-\rho_{R}\right) .
$$

If the second summand of formula (C.7) is divided by and multiplied on $\left\langle v_{f} \Sigma_{f} \cdot v \cdot \Delta n_{C+R} \cdot n_{C+R}^{+}\right\rangle_{C+R}$, then the summand transforms into the following form:

$$
\begin{aligned}
\frac{\left\langle\Delta n_{C+R}, n_{C+R}^{+}\right\rangle_{C+R}}{\left\langle v_{f} \Sigma_{f} \cdot v \cdot n_{C+R} \cdot n_{C+R}^{+}\right\rangle_{C+R}} \\
=\frac{\left\langle\Delta n_{C+R}, n_{C+R}^{+}\right\rangle_{C+R}}{\left\langle v_{f} \Sigma_{f} \cdot v \cdot \Delta n_{C+R} \cdot n_{C+R}^{+}\right\rangle_{C+R}} \\
\quad \frac{\left\langle v_{f} \Sigma_{f} \cdot v \cdot \Delta n_{C+R} \cdot n_{C+R}^{+}\right\rangle_{C+R}}{\left\langle v_{f} \Sigma_{f} \cdot v \cdot n_{C+R} \cdot n_{C+R}^{+}\right\rangle_{C+R}} .
\end{aligned}
$$

The first fraction in the right part is a mean lifetime of those neutrons, which escaped the reactor core, came back and contributed into the chain fission reaction:

$$
\bar{\Lambda}_{R} \equiv \frac{\left\langle\Delta n_{C+R}, n_{C+R}^{+}\right\rangle_{C+R}}{\left\langle\nu_{f} \Sigma_{f} \cdot v \cdot \Delta n_{C+R} \cdot n_{C+R}^{+}\right\rangle_{C+R}} .
$$

The second fraction is a share of those neutrons, which escaped the reactor core and came back, in total value of fission neutrons. In other words, this is a contribution into total reactivity caused by the presence of the neutron reflector surrounding the reactor core.

Now we can formulate the balance relationship for twozone reactor model. The relationship can link mean prompt 
neutron lifetime $\bar{\Lambda}_{\text {prt }}$ in the reactor as a whole with mean lifetime of those neutrons, which did not escape the reactor core $\left(\bar{\Lambda}_{C}\right)$, with lifetime of those neutrons, which escaped the reactor core, went into the neutron reflector, came back into the reactor core and contributed into the chain fission reaction $\left(\bar{\Lambda}_{R}\right)$, and with contribution of the neutron reflector into total reactivity $\rho_{R}$ :

$$
\bar{\Lambda}_{\mathrm{prt}}=\bar{\Lambda}_{\mathrm{C}} \cdot\left(1-\rho_{R}\right)+\bar{\Lambda}_{R} \cdot \rho_{R} .
$$

The balance relationship can be used, for example, to determine mean lifetime of those neutrons, which visited the neutron reflector $\bar{\Lambda}_{R}$.

If the neutron reflector is divided into several annular layers, then the approach presented above can be applied to determine mean lifetime of those neutrons, which visited each layer of the reflector. Thus, dependency of mean neutron lifetime on depth of neutron penetration into the reflector can be calculated. Then, the approach can be generalized for continuous model of the neutron reflector divided into infinite number of infinitesimally thin annular layers. In this case, the balance relationship for $(j+1)$ layer can be written in the following recurrent form:

$$
\Lambda_{\mathrm{prt}}^{j+1}=\Lambda_{\mathrm{prt}}^{j} \cdot\left(1-\rho_{R}^{j+1}\right)+\Lambda_{R}^{j+1} \cdot \rho_{R}^{j+1} .
$$

\section{Roots of the Inverse-Hour Equation for the Fast Reactor Core Surrounded by the Neutron Reflector-Converter}

The inverse-hour equation for the fast reactor core surrounded by the neutron reflector-converter can be written in the following form with accounting for six groups of delayed neutrons:

$$
\rho=\frac{\bar{\Lambda}_{C}}{T}+\int_{\Lambda_{\min }}^{\Lambda_{\max }} \frac{\Lambda_{R}^{\prime} \cdot\left(d \rho_{R} / d \Lambda_{R}^{\prime}\right)}{\Lambda_{R}^{\prime}+T} \cdot d \Lambda_{R}^{\prime}+\sum_{i=1}^{6} \frac{\beta_{i}}{1+\lambda_{i} \cdot T} .
$$

In this equation the lower limit of integration can be taken as $\Lambda_{\min } \approx \bar{\Lambda}_{C}$. As for the upper limit of integration $\Lambda_{\max }$, it depends on thickness and structure of the neutron reflector. For the BREST-type fast reactor core surrounded by ${ }^{208} \mathrm{~Pb}$ reflector the upper limit $\Lambda_{\max }$ can be taken from the curve $\rho_{R}\left(\Lambda_{R}\right)$ shown in Figure 2 .

For this case the roots of the inverse-hour equation (D.1) are presented in Figure 11(b). For comparison, the roots of the inverse-hour equation for the "traditional" (in a certain sense) fast reactor core surrounded by the fertile fuel breeding zone are shown in Figure 11(a).

As the neutron reflector-converter adjoins closely to the reactor core, neutron lifetime in the nearest reflector layer differs slightly from $\bar{\Lambda}_{C}$ (slightly longer because some neutrons can be reflected from the nearest layer, they undergo only a few collisions before their return to the reactor core). As is seen, the roots of the inverse-hour equation for the fast reactor core surrounded by the neutron reflector-converter covered the gap between $\bar{\Lambda}_{C}$ and the roots related with six groups of delayed neutrons.

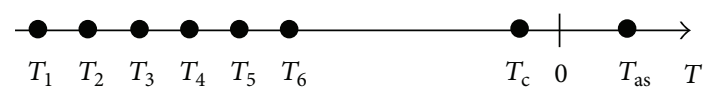

(a) Fast reactor with fertile fuel breeding zone

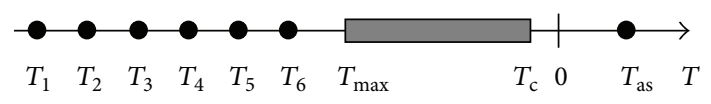

(b) Fast reactor with neutron reflector-converter

FIGURE 11: Roots of the inverse-hour equation for two types of the reflected fast reactor core $\left(T_{\mathrm{as}}\right.$-root corresponding to the asymptotic power excursion period).

D.1. On Applicability of the "Zero Prompt Neutron Lifetime" Approximation to Solving the Kinetic Equations for the Fast Reactor Core Surrounded by the Neutron ReflectorConverter. The neutron kinetics equations for the fast reactor core surrounded by the neutron reflector-converter (without accounting for any feedback effects) can be written in the following form:

$$
\begin{gathered}
\frac{d n(t)}{d t}=\frac{\rho_{0}-\beta-\rho_{R}\left(\Lambda_{\max }\right)}{\bar{\Lambda}_{C}} \cdot n(t) \\
\quad+\int_{\bar{\Lambda}_{C}}^{\Lambda_{\max }} \frac{1}{\Lambda_{R}^{\prime}} \cdot C_{R}\left(\Lambda_{R}^{\prime}, t\right) \cdot d \Lambda_{R}^{\prime}+\sum_{i=1}^{6} \lambda_{i} \cdot C_{i}(t) ; \\
\frac{d C_{i}(t)}{d t}=\beta_{i} \cdot \frac{n(t)}{\bar{\Lambda}_{C}}-\lambda_{i} \cdot C_{i}(t) ; \quad i=1, \ldots, 6 ; \\
\frac{\partial C_{R}\left(\Lambda_{R}, t\right)}{\partial t}=\frac{d \rho_{R}}{d \Lambda_{R}} \cdot \frac{n(t)}{\bar{\Lambda}_{C}}-\frac{1}{\Lambda_{R}} \cdot C_{R}\left(\Lambda_{R}, t\right) ; \\
\frac{d \rho_{R}}{d \Lambda_{R}}=\frac{d \rho_{R}(r)}{d r} \cdot \frac{d r}{d \Lambda_{R}} .
\end{gathered}
$$

As is well-known, prompt neutron lifetime in a fast reactor (FR) core is very short $\left(\bar{\Lambda}_{C} \approx 10^{-7} \div 10^{-6} \mathrm{~s}\right.$ ) while mean lifetime of delayed neutrons covers the range from $0.3 \mathrm{~s}$ to $80 \mathrm{~s}$; that is, prompt neutron lifetime is shorter than delayed neutron lifetime by 6-8 orders of magnitude. When an accidental power excursion occurs, there are two components of the time-dependent process in fast reactors: fast-acting component and slow-acting one. Numerical solution of so rigid equations is a very complicated task because correct integration of the fast-acting component and, in addition, the slow-acting component requires ultra-large number of ultrashort time steps that can lead to a significant loss of accuracy. Under these conditions, the "zero prompt neutron lifetime" approximation can be very helpful to overcome the difficulty.

Unfortunately, one else problem takes place in numerical analysis of neutron kinetics in the fast reactor core surrounded by the neutron reflector-converter. The matter is the neutrons coming back from the reflector into the reactor core and contributing into the power excursion process are characterized by lifetimes that continuously cover the range from $\bar{\Lambda}_{C}$ up to nearly the very short-lived (sixth) group 
of delayed neutrons (Figure 11). In other words, from pure mathematical point of view, it is impossible to define the fast reactor core surrounded by the neutron reflector-converter as a two-scale system with only two time-dependent fast-acting and slow-acting components.

The following specific feature must be also taken into consideration. The feature is related with time dependency of the integrand in the second summand of the inversehour equation (D.1). The integrand defines the contribution of the neutrons coming back from the reflector with various lifetimes $\Lambda_{R}$ into the reactivity gain (Figure 5 ). The time dependency allowed us to conclude that the contributions of short-lived neutrons coming back from the reflector into the reactivity gain needed to provide the power excursion with a given asymptotic period $T$ (e.g., within the range from 0.01 $\mathrm{s}$ to $10 \mathrm{~s}$ ) would be relatively small. This circumstance opens an opportunity to obtain a sufficiently correct upper evaluation of the power excursion by introducing the following additional assumption. Let us assume that if the neutrons coming back from the reflector are characterized by lifetimes shorter than a certain value $\left(\Lambda_{0}=10^{-4} \mathrm{~s}\right.$, for instance), then a new lifetime, namely, neutron lifetime in the reactor core $\bar{\Lambda}_{C}$, is given to these neutrons. Under these assumptions the neutron kinetics equations can be written as follows:

$$
\begin{gathered}
\frac{d n(t)}{d t}=\frac{\rho_{0}-\beta-\left[\rho_{R}\left(\Lambda_{\max }\right)-\rho_{R}\left(\Lambda_{0}\right)\right]}{\bar{\Lambda}_{C}} \cdot n(t) \\
\quad+\int_{\Lambda_{0}}^{\Lambda_{\max }} \frac{1}{\Lambda_{R}^{\prime}} \cdot C_{R}\left(\Lambda_{R}^{\prime}, t\right) \cdot d \Lambda_{R}^{\prime}+\sum_{i=1}^{6} \lambda_{i} \cdot C_{i}(t) ; \\
\frac{d C_{i}(t)}{d t}=\beta_{i} \cdot \frac{n(t)}{\bar{\Lambda}_{C}}-\lambda_{i} \cdot C_{i}(t) ; \quad i=1, \ldots, 6 ; \\
\frac{\partial C_{R}\left(\Lambda_{R}, t\right)}{\partial t}=\frac{d \rho_{R}}{d \Lambda_{R}} \cdot \frac{n(t)}{\bar{\Lambda}_{C}}-\frac{1}{\Lambda_{R}} \cdot C_{R}\left(\Lambda_{R}, t\right) .
\end{gathered}
$$

The inverse-hour equation that links the reactivity gain with the required asymptotic power excursion period $T$ transforms into the following form:

$$
\rho=\frac{\bar{\Lambda}_{C}}{T}+\int_{\Lambda_{0}}^{\Lambda_{\max }} \frac{\Lambda_{R}^{\prime} \cdot\left(d \rho_{R} / d \Lambda_{R}^{\prime}\right)}{\Lambda_{R}^{\prime}+T} \cdot d \Lambda_{R}^{\prime}+\sum_{i=1}^{6} \frac{\beta_{i}}{1+\lambda_{i} \cdot T}
$$

The roots of the equation can be presented in Figure 12.

By using the standard mathematical operations (described in [1], e.g.), the "zero prompt neutron lifetime" approximation can be obtained for system of equations (D.3):

$$
\begin{aligned}
\frac{n(t)}{\bar{\Lambda}_{C}}= & \frac{(-1)}{\rho_{0}-\beta-\left[\rho_{R}\left(\Lambda_{\max }\right)-\rho_{R}\left(\Lambda_{0}\right)\right]} \\
& \cdot\left[\int_{\Lambda_{0}}^{\Lambda_{\max }} \frac{1}{\Lambda_{R}^{\prime}} C_{R}\left(\Lambda_{R}^{\prime}, t\right) \cdot d \Lambda_{R}^{\prime}+\sum_{i=1}^{6} \lambda_{i} C_{i}(t)\right] ;
\end{aligned}
$$

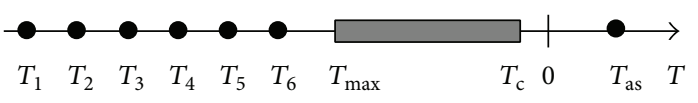

(a) FR with the neutron reflector-converter

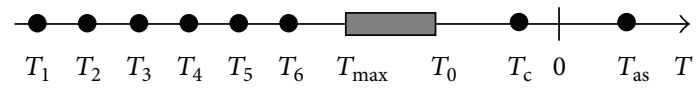

(b) FR with the neutron reflector-converter (the neutrons coming back from the reflector with lifetimes $\Lambda_{R}<\Lambda_{0}$ are united with the neutrons in the reactor core)

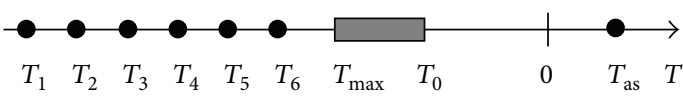

(c) Item "b" (plus the "zero prompt neutron lifetime" in the reactor core)

FIGURE 12: Roots of the inverse-hour equation for FR with the neutron reflector-converter $\left(T_{\mathrm{as}}\right.$-root corresponding to the asymptotic power excursion period).

$$
\begin{aligned}
& \frac{d C_{i}(t)}{d t}+\lambda_{i} \cdot C_{i}(t)=\beta_{i} \cdot \frac{n(t)}{\bar{\Lambda}_{C}} ; \quad i=1, \ldots, 6 ; \\
& \frac{\partial C_{R}\left(\Lambda_{R}, t\right)}{\partial t}+\frac{1}{\Lambda_{R}} \cdot C_{R}\left(\Lambda_{R}, t\right)=\frac{d \rho_{R}}{d \Lambda_{R}} \cdot \frac{n(t)}{\bar{\Lambda}_{C}} .
\end{aligned}
$$

The transformed system (D.5) can be solved much easier than initial system (D.3) because of the following reasons:

(i) system (D.5) does not contain differential equation for neutron flux $n(t)$;

(ii) time scale (lifetimes of neutrons and emitters of delayed neutrons) now covers a relatively longer range (from $10^{-4} \mathrm{~s}$ to $10 \mathrm{~s}$ ).

Solution of system (D.5) can be found by using an iterative process which is simplified by the fact that the second and third equations can be solved analytically:

$$
\begin{gathered}
\frac{d y}{d t}+\lambda \cdot y=A(t) \\
y(t)=y(0) \cdot e^{-\lambda \cdot t}+\int_{0}^{t} d t^{\prime} \cdot e^{-\lambda\left(t-t^{\prime}\right)} \cdot A\left(t^{\prime}\right) .
\end{gathered}
$$

The following feature in time dependency of neutron flux $n(t)$ must be noted. At $t=0$ neutron flux undergoes an ordinary jump; that is, $n(t=+0)=n(t=-0)+\Delta n(t=0)$, where $t= \pm 0$ means $t= \pm \varepsilon$ with infinitesimal positive $\varepsilon \rightarrow 0$. At the same time, concentrations of delayed neutron emitters remain continuous functions; that is, $C_{i}(t=-0)=C_{i}(t=$ $+0)$.

As to the function $C_{R}(\Lambda, t)$ defining pseudo neutron emitters in the reflector, taking into account relatively rapid migration of prompt fission neutrons from the reactor core into the reflector (the migration time is usually shorter than $10^{-4} \mathrm{~s}$ [27]), initial condition for the function $C_{R}(\Lambda, t)$ must 
be formed quite similarly to initial condition for neutron flux with an ordinary jump at $t=0$ :

$$
\frac{C_{R}\left(\Lambda_{R}, t=+0\right)}{C_{R}\left(\Lambda_{R}, t=-0\right)}=\frac{n(t=+0)}{n(t=-0)} .
$$

D.2. Feedbacks Accountability. Depending on the power excursion rate, some feedbacks can obtain a long enough time interval to change evolution of the process. Doppler-effect, for example, can change resonance neutron absorption at fuel warming up instantly. On the contrary, some time interval must elapse before the coolant density effect caused by its warming up actuates (heat must have a time to flow down from fuel to coolant). Thermal constant of fuel rods $\tau_{\text {therm }}$ is usually applied as a time parameter defining the process of heat flowing down $[16,28]$.

Thermal constant $\tau_{\text {therm }}$ of cylindrical fuel rods with oxide fuel meat depends on their diameter and can vary within the range of $1 s \div 3 \mathrm{~s}$ [16]. In the case of metal uranium fuel, thermal constant $\tau_{\text {therm }}$ shortens by one order of magnitude $\left(\tau_{\text {therm }} \approx 0.1 \mathrm{~s}\right)$. Thus, heat from metal fuel rods flows down substantially quicker thanks to the better heat conductivity of metal fuel. The shortest thermal constants can be achieved in micro coated-fuel particles dispersed in inert matrix $\left(\tau_{\text {therm }}=\right.$ $0.01 \mathrm{~s} \div 0.03 \mathrm{~s}$ ) [29]. The concept of micro fuel particles is under development now for advanced light-water power reactors.

So, it can be a priori stated that, if the asymptotic period $T$ of the power excursion caused by positive reactivity jump belongs to the time range of $0.01 \mathrm{~s} \div 10 \mathrm{~s}$, then the coolantinduced reactivity feedback has a long enough time interval to influence remarkably on the power excursion process. This, in its turn, allows us to consider the question: what positive reactivity jumps are acceptable for the feedbacks could actuate?

D.3. Fast Power Excursion with Accounting for Doppler-Effect in Fuel (No Heat Flows Down to Coolant). Neutron kinetics equations in the case of fast power excursion with accounting for Doppler-effect caused by fuel warming up can be written in the following form for the FR core surrounded by the neutron reflector-converter:

$$
\begin{aligned}
& \frac{d n(t)}{d t} \\
& =\frac{\rho_{0}-\beta-\left[\rho_{R}\left(\Lambda_{\max }\right)-\rho_{R}\left(\Lambda_{0}\right)\right]-\alpha_{T} \int_{0}^{t} n\left(t^{\prime}\right) \cdot d t^{\prime}}{\bar{\Lambda}_{C}} \cdot n(t) \\
& +\int_{\Lambda_{0}}^{\Lambda_{\max }} \frac{1}{\Lambda_{R}^{\prime}} \cdot C_{R}\left(\Lambda_{R}^{\prime}, t\right) \cdot d \Lambda_{R}^{\prime}+\sum_{i=1}^{6} \lambda_{i} \cdot C_{i}(t) ; \\
& \quad \frac{d C_{i}(t)}{d t}+\lambda_{i} \cdot C_{i}(t)=\beta_{i} \cdot \frac{n(t)}{\bar{\Lambda}_{C}} ; \quad i=1, \ldots, 6 ; \\
& \quad \frac{\partial C_{R}\left(\Lambda_{R}, t\right)}{\partial t}+\frac{1}{\Lambda_{R}} \cdot C_{R}\left(\Lambda_{R}, t\right)=\frac{d \rho_{R}}{d \Lambda_{R}} \cdot \frac{n(t)}{\bar{\Lambda}_{C}},
\end{aligned}
$$

where $\rho_{0}$ is initial reactivity jump; $\int_{0}^{t} n\left(t^{\prime}\right) d t^{\prime}$ is heat accumulated in fuel by time moment $t ; \alpha_{T}$ is reactivity coefficient of Doppler-effect.

According to the well-known Nordheim-Fuchs-Hansen (NFH) model [1], thermal energy yield from the prompt power excursion with accounting for the heat accumulation in fuel and actuation of the reactivity feedback caused by Doppler-effect can be evaluated by using the following equation:

$$
\frac{d n(t)}{d t}=\frac{\rho_{0}-\beta-\alpha_{T} \int_{0}^{t} n\left(t^{\prime}\right) \cdot d t^{\prime}}{\bar{\Lambda}_{C}} \cdot n(t) .
$$

The model presented here differs from the NFH-model by the following features.

(i) Effective fraction of delayed neutrons $\beta$ is replaced by the larger value $\widehat{\beta}=\beta+\left[\rho_{R}\left(\Lambda_{\max }\right)-\rho_{R}\left(\Lambda_{0}\right)\right]$ thanks to the neutrons coming back from the reflector into the reactor core.

(ii) The right part of the first equation contains new summands which define the neutrons coming back from the reflector and delayed neutrons from decays of their emitters. These summands must be added because the time range for the procrastinated return of neutrons from the reflector stretches from neutron lifetime in the reactor core up to lifetime of the most short-lived group of delayed neutrons.

(iii) System of neutron kinetics equations must contain equations for the neutrons coming back from the reflector and for delayed neutrons.

The approach described in [1] for solving the NFHequations can be also used to transform the first equation of system (D.8) for time evolution of neutron flux $n(t)$. At first, the following designation is introduced and differentiated with respect to time:

$$
\begin{gathered}
y(t) \equiv\left[\rho_{0}-\widehat{\beta}-\alpha_{T} \int_{0}^{t} n\left(t^{\prime}\right) \cdot d t^{\prime}\right], \\
\frac{d y(t)}{d t}=-\alpha_{T} \cdot n(t) ; \\
y(t) \cdot \frac{d y(t)}{d t}=-\left(\alpha_{T} \cdot \bar{\Lambda}_{C}\right) \cdot\left[\frac{y(t)}{\bar{\Lambda}_{C}} \cdot n(t)\right] .
\end{gathered}
$$

Then, application of the "zero prompt neutron lifetime" approximation to the first equation of system (D.8) results in the following integral equation for $\left(y(t) / \bar{\Lambda}_{C}\right) \cdot n(t)$ :

$$
\begin{aligned}
& {\left[\frac{y(t)}{\bar{\Lambda}_{C}} \cdot n(t)\right]} \\
& \quad=-\left\{\int_{\Lambda_{0}}^{\Lambda_{\max }} \frac{1}{\Lambda_{R}^{\prime}} \cdot C_{R}\left(\Lambda_{R}^{\prime}, t\right) \cdot d \Lambda_{R}^{\prime}+\sum_{i=1}^{6} \lambda_{i} \cdot C_{i}(t)\right\} .
\end{aligned}
$$


Minus in the right part means that function $y(t)$ is negative; that is,

$$
\rho_{0}<\widehat{\beta}=\beta+\left[\rho_{R}\left(\Lambda_{\max }\right)-\rho_{R}\left(\Lambda_{0}\right)\right]
$$

In other words, initial reactivity jump must not exceed fraction of the neutrons which can contribute to the chain fission reaction after a certain time delay only. These neutrons include the following two components: delayed (in a traditional sense) neutrons and prompt (by their origination) neutrons coming back from the reflector with a certain time delay. If expression (D.11) is substituted into (D.10), then

$$
\begin{aligned}
y(t) \cdot \frac{d y(t)}{d t}=+ & \left(\alpha_{T} \cdot \bar{\Lambda}_{C}\right) \\
& \cdot\left\{\int_{\Lambda_{0}}^{\Lambda_{\max }} \frac{1}{\Lambda_{R}^{\prime}} \cdot C_{R}\left(\Lambda_{R}^{\prime}, t\right) \cdot d \Lambda_{R}^{\prime}\right. \\
& \left.+\sum_{i=1}^{6} \lambda_{i} \cdot C_{i}(t)\right\} .
\end{aligned}
$$

Some simple mathematical transformations of the left part allowed us to find solution of (D.13) in quadratures:

$$
\begin{aligned}
\frac{1}{2} \frac{d y^{2}}{d t}= & +\left(\alpha_{T} \cdot \bar{\Lambda}_{C}\right) \\
& \cdot\left\{\int_{\Lambda_{0}}^{\Lambda_{\max }} \frac{1}{\Lambda_{R}^{\prime}} \cdot C_{R}\left(\Lambda_{R}^{\prime}, t\right) \cdot d \Lambda_{R}^{\prime}+\sum_{i=1}^{6} \lambda_{i} \cdot C_{i}(t)\right\} ; \\
y^{2}(t)=2 & \cdot\left(\alpha_{T} \cdot \bar{\Lambda}_{C}\right) \\
& \times \int_{0}^{t} d t^{\prime}\left\{\int_{\Lambda_{0}}^{\Lambda_{\max }} \frac{1}{\Lambda_{R}^{\prime}} C_{R}\left(\Lambda_{R}^{\prime}, t^{\prime}\right) \cdot d \Lambda_{R}^{\prime}\right. \\
& \left.+\sum_{i=1}^{6} \lambda_{i} \cdot C_{i}\left(t^{\prime}\right)\right\}+ \text { const. }
\end{aligned}
$$

Initial condition $y^{2}(0)=\left(\rho_{0}-\widehat{\beta}\right)^{2}$ can be used to obtain the following relationship: const $=\left(\rho_{0}-\widehat{\beta}\right)^{2}$. Then,

$$
\begin{aligned}
y(t)=-\left\{2 \cdot\left(\alpha_{T} \cdot \bar{\Lambda}_{C}\right)\right. & \\
& \times \int_{0}^{t} d t^{\prime}\left[\int_{\Lambda_{0}}^{\Lambda_{\max }} \frac{1}{\Lambda_{R}^{\prime}} C_{R}\left(\Lambda_{R}^{\prime}, t^{\prime}\right) d \Lambda_{R}^{\prime}\right. \\
& \left.\left.+\sum_{i=1}^{6} \lambda_{i} C_{i}\left(t^{\prime}\right)\right]+\left(\rho_{0}-\widehat{\beta}\right)^{2}\right\}^{1 / 2} .
\end{aligned}
$$

Now we obtained the following equations for time evolution of neutron flux, emitters of delayed neutrons and pseudoemitters of the neutrons coming back from the reflector:

$$
\begin{gathered}
{\left[\frac{n(t)}{\bar{\Lambda}_{C}}\right]=\frac{(-1)}{y(t)}} \\
\cdot\left\{\int_{\Lambda_{0}}^{\Lambda_{\max }} \frac{1}{\Lambda_{R}^{\prime}} \cdot C_{R}\left(\Lambda_{R}^{\prime}, t\right) \cdot d \Lambda_{R}^{\prime}+\sum_{i=1}^{6} \lambda_{i} \cdot C_{i}(t)\right\} ; \\
\frac{d C_{i}(t)}{d t}+\lambda_{i} \cdot C_{i}(t)=\beta_{i} \cdot\left[\frac{n(t)}{\bar{\Lambda}_{C}}\right] ; \quad i=1, \ldots, 6 ; \\
\frac{\partial C_{R}\left(\Lambda_{R}, t\right)}{\partial t}+\frac{1}{\Lambda_{R}} \cdot C_{R}\left(\Lambda_{R}, t\right)=\frac{d \rho_{R}}{d \Lambda_{R}} \cdot\left[\frac{n(t)}{\bar{\Lambda}_{C}}\right]
\end{gathered}
$$

This system of integral and differential equations can be transformed into system of integral equations by using analytical expression (D.6):

$$
\begin{aligned}
{\left[\frac{n(t)}{\bar{\Lambda}_{C}}\right]=} & \frac{(-1)}{y(t)} \cdot\left\{\int_{\Lambda_{0}}^{\Lambda_{\max }} \frac{1}{\Lambda_{R}^{\prime}} \cdot C_{R}\left(\Lambda_{R}^{\prime}, t\right) \cdot d \Lambda_{R}^{\prime}\right. \\
& \left.+\sum_{i=1}^{6} \lambda_{i} \cdot C_{i}(t)\right\} ; \\
C_{i}(t)= & C_{i}(t=+0) \cdot e^{-\lambda_{i} t} \\
& +\beta_{i} \cdot \int_{0}^{t} d t^{\prime} \cdot e^{-\lambda_{i}\left(t-t^{\prime}\right)} \cdot\left[\frac{n\left(t^{\prime}\right)}{\bar{\Lambda}_{C}}\right] ; \\
C_{R}\left(\Lambda_{R}, t\right)= & C_{R}\left(\Lambda_{R}, t=+0\right) \\
& \cdot e^{-t / \Lambda_{R}}+\frac{d \rho_{R}}{d \Lambda_{R}} \cdot \int_{0}^{t} d t^{\prime} \cdot e^{-\left(1 / \Lambda_{R}\right)\left(t-t^{\prime}\right)} \\
& \cdot\left[\frac{n\left(t^{\prime}\right)}{\bar{\Lambda}_{C}}\right] \cdot
\end{aligned}
$$

This nonlinear system of integral equations can be solved by an iterative method.

\section{Conflict of Interests}

The authors declare that there is no conflict of interests regarding the publication of this paper.

\section{References}

[1] G. J. Bell and S. Glasstone, Nuclear Reactor Theory, Van Nostrand Reinhold, New York, NY, USA, 1970.

[2] A. N. Shmelev, G. G. Kulikov, V. B. Glebov, D. F. Tsurikov, and A. G. Morozov, "Safety in a fast burning reactor for long-lived actinides extracted from radioactive wastes," Atomic Energy, vol. 73, no. 6, pp. 963-966, 1992.

[3] A. N. Shmelev, G. G. Kulikov, V. A. Apse, V. B. Glebov, D. F. Tsurikov, and A. G. Morozov, "Radiowaste transmutation in 
nuclear reactors," in Proceedings of the Specialists Meeting Use of Fast Reactors for Actinide Transmutation, IAEA-TECHDOC693, pp. 77-86, IAEA, Obninsk, Russia, September 1993.

[4] G. G. Kulikov, V. A. Apse, E. G. Kulikov, A. N. Shmelev, and V. A. Yufereva, "On nuclear-physical potential of radiogenic lead," in Proceedings of the 15th Seminar Volga: Actual Problems of Nuclear Reactors Physics-Efficiency, Safety, Nonproliferation, pp. 141-144, Moscow, Russia, September 2008.

[5] A. N. Shmelev, G. G. Kulikov, V. A. Apse, E. G. Kulikov, and V. V. Artisyuk, "Radiogenic lead with dominant content of ${ }^{208} \mathrm{~Pb}$ : new coolant and neutron moderator for innovative nuclear facilities," Science and Technology of Nuclear Installations, vol. 2011, Article ID 252903, 12 pages, 2011.

[6] G. G. Kulikov, A. N. Shmelev, V. A. Apse, and E. G. Kulikov, "Lead, containing isotope $\mathrm{Pb}-208$ - heavy neutron moderator and reflector. Its neutron-physical properties," in Proceedings of the Scientific Session of NRNU MEPhI, vol. 1, p. 49, Moscow, Russia, January 2011.

[7] A. N. Shmelev, E. G. Kulikov, G. G. Kulikov, and V. A. Apse, "Lead containing mainly isotope ${ }^{208} \mathrm{~Pb}$ - a neutron moderator, coolant and reflector of neutrons. Its neutron-physical properties," in Proceedings of the 10th International Conference toward and over the Fukushima Daiichi Accident (GLOBAL '11), Makuhari, Japan, December 2011.

[8] V. V. Orlov, A. I. Filin, A. V. Lopatkin et al., "The closed on-site fuel cycle of the BREST reactors," Progress in Nuclear Energy, vol. 47, no. 1-4, pp. 171-177, 2005.

[9] V. F. Kolesov, Aperiodical Pulsed Reactors, RFNC-VNIIEF, Sarov, Russia, 2007.

[10] G. D. Spriggs and R. D. Bush, "Reflected reactors: point kinetics and prompt critical," in Proceedings of the Topical Meeting on Physics, Safety, and Applications of Pulse Reactors, pp. 265-273, Washington, DC, USA, November 1994.

[11] G. D. Spriggs, R. D. Bush, and J. G. Williams, "Two-region kinetic models for reflected reactors," Annals of Nuclear Energy, vol. 1, no. 3, 1996.

[12] C. E. Cohn, "Reflected-reactor kinetics," Nuclear Science and Engineering, vol. 13, no. 1, 1962.

[13] D. P. Gamble, "The effect of reflector-moderated neutrons on the kinetics of the kinetic experiment water boiler," Transactions of American Nuclear Society, vol. 3, no. 1, p. 122, 1960.

[14] R. L. Coats and R. L. Long, "Reflector and decoupling experiments with fast burst reactors," in Proceedings of the National Topical Meeting on Fast Burst Reactors, Albuquerque, NM, USA, January 1969.

[15] R. Avery, "Theory of decoupled reactors," in Proceedings of the 2nd International Conference Peaceful Uses of Atomic Energy, vol. 12, pp. 182-191, Geneva, Switzerland, 1958.

[16] H. H. Hummel and D. Okrent, Reactivity Coefficients in Large Fast Power Reactors, American Nuclear Society, LaGrange Park, Ill, USA, 1970.

[17] K. Shibata, O. Iwamoto, T. Nakagawa et al., "JENDL-4.0: a new library for nuclear science and engineering," Journal of Nuclear Science and Technology, vol. 48, no. 1, pp. 1-30, 2011.

[18] I. S. Grigoriev and E. Z. Meylikhov, Physical Values. Reference Book, Energoatomizdat, Moscow, Russia, 1991.

[19] A. D. Galanin, Introduction to a Theory of Thermal Nuclear Reactors, Energoatomizdat, Moscow, Russia, 1990.

[20] J. M. Godoy, M. L. D. P. Godoya, and C. C. Aronne, "Application of inductively coupled plasma quadrupole mass spectrometry for the determination of monazite ages by lead isotope ratios,"
Journal of the Brazilian Chemical Society, vol. 18, no. 5, pp. 969975, 2007.

[21] A. O. Nier, R. W. Thompson, and B. F. Murphey, "The isotopic constitution of lead and the measurement of geological time," Physical Review, vol. 60, no. 2, pp. 112-116, 1941.

[22] A. Holmes, "The pre-cambrian and associated rocks of the district of Mozambique," Quarterly Journal of the Geological Society, vol. 74, no. 1-4, pp. 31-98, 1918.

[23] T. C. Sarkar, "The lead ratio of a crystal of monazite from the Gaya district, Bihar," Proceedings of the Indian Academy of Sciences Section A, vol. 13, no. 3, pp. 245-248, 1941.

[24] Catalog of Isotope Dates for Minerals of Ukrainian Shield, Naukova Dumka, Kiev, Ukraine, 1978.

[25] N. M. Sinev, Economics of Nuclear Power, Energoatomizdat, Moscow, Russia, 1987.

[26] J. A. Seneda, C. A. L. G. de O. Forbicini, C. A. D. S. Queiroz et al., "Study on radiogenic lead recovery from residues in thorium facilities using ion exchange and electrochemical process," Progress in Nuclear Energy, vol. 52, no. 3, pp. 304-306, 2010.

[27] K. H. Beckurts and K. Wirtz, Neutron Physics, Springer, Berlin, Germany, 1964.

[28] I. A. Kuznetsov and V. M. Poplavsky, Safety of NPP with Fast Reactors, IZDAT, Moscow, Russia, 2012.

[29] N. N. Ponomarev-Stepnoj, N. E. Kukharkin, A. A. Khrulev et al., "Prospects of coated fuel particle application in the WWER reactors," Atomnaya Energiya, vol. 86, no. 6, pp. 443-449, 1999. 


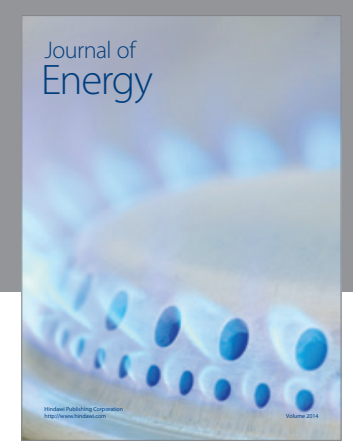

Journal of

Industrial Engineering
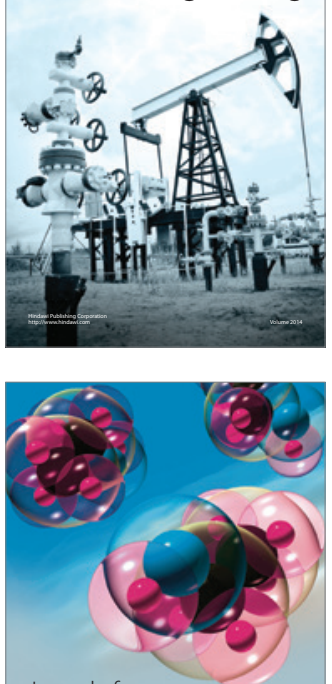

Fuels
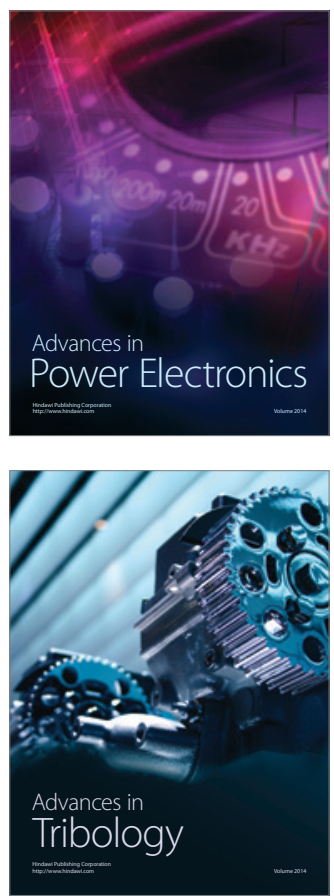

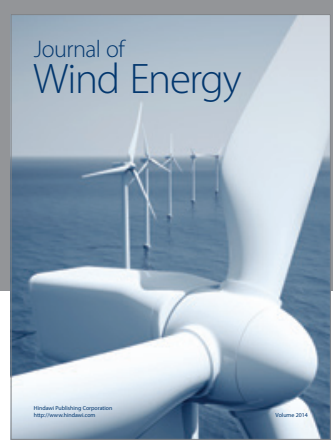

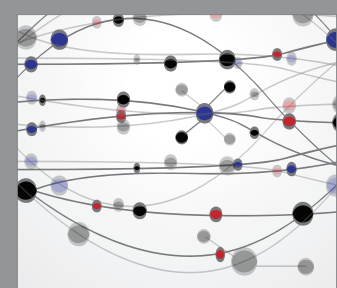

The Scientific World Journal

Submit your manuscripts at http://www.hindawi.com

Journal of

Structures
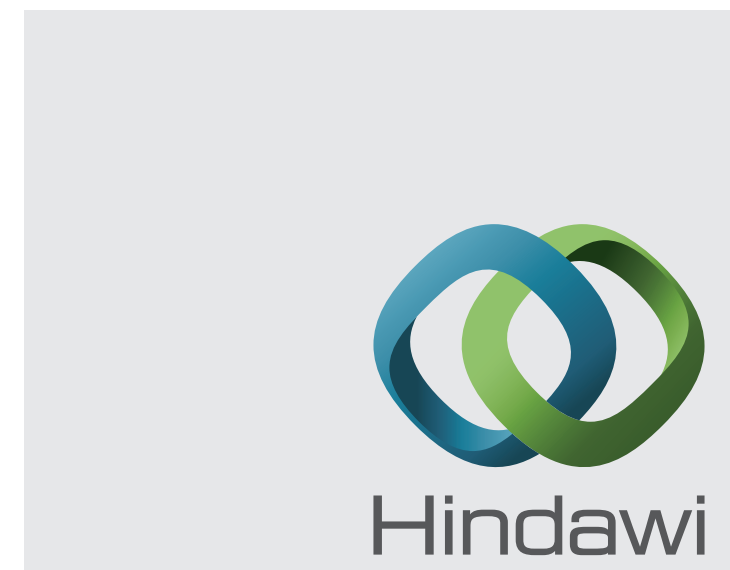

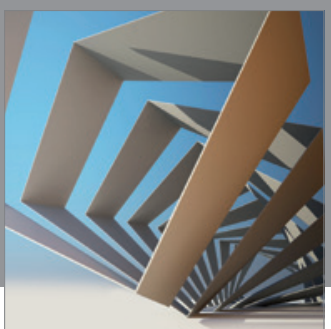

Rotating

Machinery
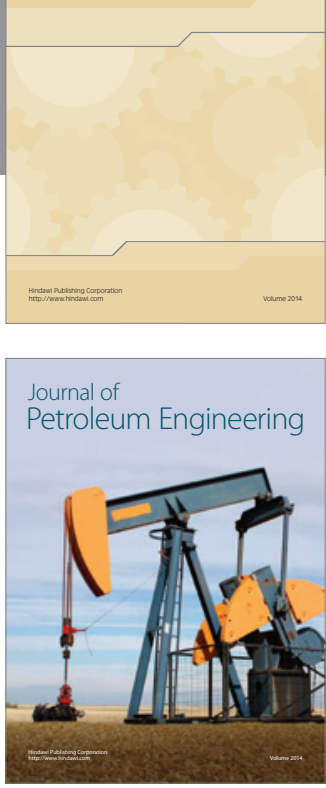

Journal of

Solar Energy
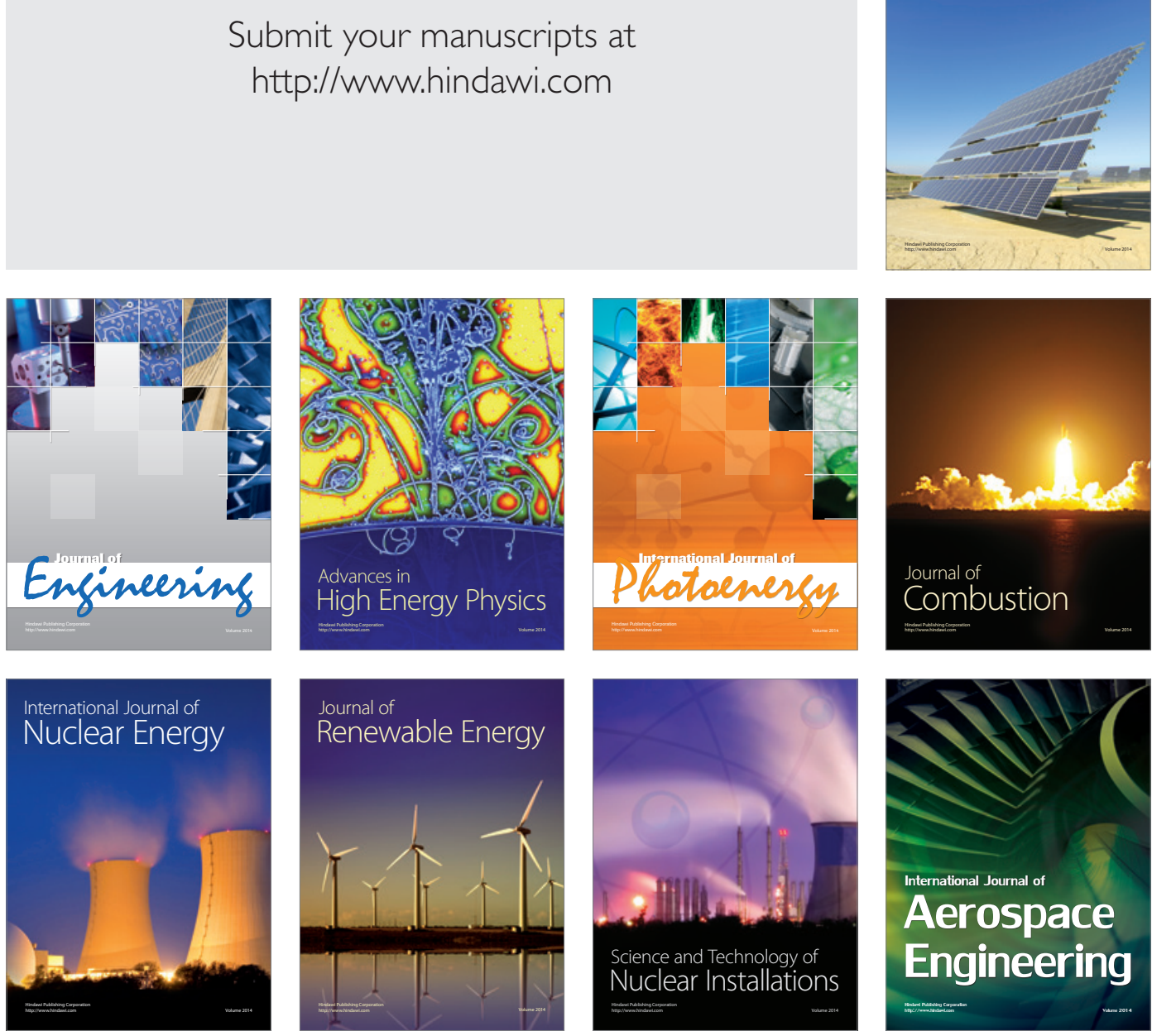